\title{
VORTEX DYNAMO IN AN OBLIQUELY ROTATING STRATIFIED NANOFLUID BY SMALL-SCALE NON-HELICAL FORCES
}

\author{
Dichael I. Kopp ${ }^{a *}$, (D)Anatoly V. Tur ${ }^{c}$, Volodymyr V. Yanovsky ${ }^{a, b}$ \\ ${ }^{a}$ Institute for Single Cristals, Nat. Academy of Science Ukraine \\ Nauky Ave. 60, Kharkov 31001, Ukraine \\ ${ }^{\mathrm{b}}$ V.N. Karazin Kharkiv National University \\ 4, Svoboda Sq., Kharkiv, 61022, Ukraine \\ ${ }^{c}$ Universite Toulouse [UPS], CNRS, Institute of Research for Astrophysics and Planetology \\ 9 avenue du Colonel Roche, BP 44346, 31028 Toulouse Cedex 4, France \\ *Corresponding Author: michaelkopp0165@gmail.com \\ Received February 9, 2021; revised March 11, 2021; accepted April 9, 2021
}

In this work, a large-scale instability of the hydrodynamic $\alpha$-effect in an obliquely rotating stratified nanofluid taking into account the effects of Brownian diffusion and particle flux under the influence of a temperature gradient (thermophoresis) is obtained. The instability is caused by the action of an external small-scale non-spiral force, which excites small-scale velocity oscillations with zero helicity and a low Reynolds number. Nonlinear equations for large-scale motions are obtained using the method of multiscale asymptotic expansions by a small parameter (Reynolds number). A linear large-scale instability of hydrodynamic $\alpha$-effect is investigated depending on the parameters of rotation $D$, temperature stratification $\widetilde{R a}$, and concentration of nanoparticles $\widetilde{R}_{n}$. A new effect of the generation of large-scale vortex structures in nanofluid at $\widetilde{R a}=0$ is associated with an increase in the concentration of nanoparticles is obtained. The maximum instability increment is reached at inclination angles $\theta \approx \pi / 5$ for the Prandtl numbers $\operatorname{Pr}=5$, and for the Prandtl numbers $\operatorname{Pr}=1$ at inclination angles $\theta \approx \pi / 2$. It has been found that the frequency changing of the parametric impact will make it possible to control and track the generation of large-scale vortex structures. It is shown that circularly polarized Beltrami vortices appear in nanofluid as the result of new large-scale instability development. In this paper, the saturation regime of large-scale instability in an obliquely rotating stratified nanofluid with an external small-scale non-spiral force is investigated. In the stationary regime was obtained a dynamic system of equations for large-scale perturbations of the velocity field. Numerical solutions of this system of equations are obtained, which show the existence of localized vortex structures in the form of nonlinear Beltrami waves and kinks. The velocity profile of kink tends to be constant at large $\mathrm{Z}$ values.

KEY WORDS: stratified nanofluid, large-scale instability, Coriolis force, multiscale asymptotic expansions, $\alpha$-effect, localized vortex structures

In recent years there has been considerable attention to the problem of transfer processes in nanofluids, which describe a two-phase system consisting of a carrier medium (base fluid or gas) and nanoparticles with characteristic sizes from 1 to $100 \mathrm{~nm}$ [1]. Typical base fluids are water or some organic fluids, polymer solutions etc., and nanoparticles are usually particles of metals $(\mathrm{Al}, \mathrm{Cu})$, metal oxides $\left(\mathrm{A} l_{2} \mathrm{O}_{3}, \mathrm{CuO}\right)$. It is obvious that the effective use of nanofluids will become possible only when carrying out the theoretical studies of transfer processes. Special attention should be paid to the processes of formation and evolution of large-scale structures that affect heat transfer in nanofluids.

In works [2-4] were investigated conditions for convective structures (convective cells) formation in rotating layers of nanofluids taking into account the effects of thermophoresis and Brownian motion of particles. In [2] it was shown that the temperature gradient and rotation have a stabilizing effect, while the volume fraction of nanoparticles and the ratio of nanoparticles density to the base fluid have a destabilizing effect on the system. In [3] was carried out a weakly nonlinear analysis of stability in a horizontal rotating nanofluid layer using the minimum order of the Fourier series expansion. The values of heat transfer $N u$ and transfer of nanoparticle concentration $N u_{\varphi}$ oscillate with time initially strongly but with time reach a stationary value. The parameters of the Rayleigh number concentration $R_{n}$ and the Lewis number $L_{e}$ increase the heat and mass transfer rates. An increase of the rotation parameter (Taylor number) leads to a decrease of the heat and mass transfer rates. In paper [4] was investigated the effect of variable gravity on the occurrence of thermal convection in a horizontal layer of rotating nanofluid for a porous medium. It was also shown there that a decrease in the gravity parameter has a stabilizing effect, while an increase in the gravity parameter has a destabilizing effect on the stationary convection. In this case, the critical Rayleigh number increases with an increase of the Taylor number, which indicates the suppression of the onset of convection. The influence of a periodic external action leads to the formation of oscillate convective structures. Therefore, it becomes possible to control the process of heat transfer through the cells, i.e., in the heat valve mode. Here it becomes possible to control the switching between high and low thermal conductivity modes of the cell [5].

Unlike preceding articles [2-5] in this paper we investigated the large-scale vortex structures (LSVS) formation mechanism in a rotating layer of stratified nanofluid under the influence of an external small-scale force. The generation 
of LSVS in a Newtonian rotating and temperature stratified fluid under the action of a small-scale force with zero helicity $\vec{F}_{0} r o t \vec{F}_{0}=0$ was considered in [6]. A necessary condition for the occurrence of large-scale instability considered in [6] is the oblique rotation of the fluid.

The aim of this work is to study generation and nonlinear evolution of vortices fields in a rotating stratified nanofluid under the action of the nonhelical force $\vec{F}_{0}$. Similartly to study [6], we also will use a mathematical formalism based on the asymptotic method of multiscale expansions in the small parameter of the Reynolds number $R=\frac{v_{0} t_{0}}{\lambda_{0}} \ll 1[7]$.

\section{PROBLEM STATEMENT AND BASIC EVOLUTION EQUATIONS}

We consider an infinite horizontal layer of incompressible nanofluid, which rotates with constant angular velocity $\vec{\Omega}=\left(\Omega_{1}, \Omega_{2}, \Omega_{3}\right)$. The vector of angular velocity of rotation $\vec{\Omega}$ is inclined concerning to the plane $(X, Y)$ as shown in Fig. 1.

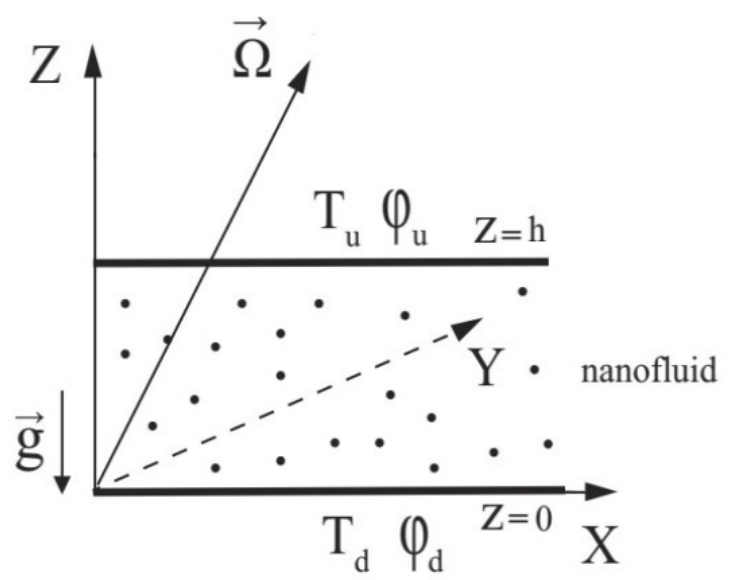

Figure 1. The angular velocity $\vec{\Omega}$ is inclined to the plane $(X, Y)$ where the external force $\vec{F}_{0}$ is located.

The nanofluid is enclosed between two parallel planes $z=0$ and $z=h$, where the temperature and volume fraction of nanoparticles are kept constant:

$$
\begin{aligned}
& T=T_{d}, \varphi=\varphi_{d} \quad \text { at } \quad z=0, \\
& T=T_{u}, \varphi=\varphi_{u} \quad \text { at } \quad z=h,
\end{aligned}
$$

here $T_{d}>T_{u}, \varphi_{u}>\varphi_{d}$. We assume that the both boundaries surfaces are free. The hydrodynamic equations of a viscous incompressible rotating nanofluid in the Boussinesq approximation has the following form (see for example [2-4]):

$$
\begin{gathered}
\rho_{00}\left(\frac{\partial \vec{V}}{\partial t}+\vec{V} \cdot \nabla \vec{V}\right)=-\nabla P+\mu \nabla^{2} \vec{V}+\left[\varphi \rho_{p}+(1-\varphi) \rho_{00}\left(1-\beta\left(T-T_{u}\right)\right)\right] \vec{g}+2 \rho_{00} \vec{V} \times \vec{\Omega}+\overrightarrow{F_{0}} \\
(\rho c)_{f}\left(\frac{\partial T}{\partial t}+\vec{V} \cdot \nabla T\right)=k_{f} \nabla^{2} T+(\rho c)_{p}\left(D_{B} \nabla \varphi \cdot \nabla T+D_{T} \frac{\nabla T \cdot \nabla T}{T_{u}}\right) \\
\frac{\partial \varphi}{\partial t}+\vec{V} \cdot \nabla \varphi=D_{B} \nabla^{2} \varphi+\frac{D_{T}}{T_{u}} \nabla^{2} T \\
\nabla \vec{V}=0
\end{gathered}
$$

Equations (2)-(5) are supplemented with boundary conditions for the velocity of nanofluid motion. The condition of impermeability of layer boundaries in the vertical direction and the absence of shear stresses at the boundaries of the layer give the following boundary conditions for the velocity

$$
V_{z}=0, \quad \frac{\partial^{2} V_{z}}{\partial z^{2}}=0, \quad \text { at } \quad z=0, h
$$


Here $\mu$ is the viscosity of nanofluid, $\rho_{00}=\varphi \rho_{p}+(1-\varphi) \rho_{f}$ is the nanofluid density at the reference temperature $T_{u}$, $\rho_{p}$ is the density of the nanoparticles, $\rho_{f}$ is the base fluid density at the reference temperature $T_{u}, \varphi$ is the volumetric fraction of nanoparticles, $\beta$ is the thermal expansion coefficient, $\vec{e}=(0,0,1)$ is a unit vector in the direction of the axis $O Z, \vec{g}$ is the gravitational acceleration vector directed along the $Z$ axis: $\vec{g}=(0,0,-g) .(\rho c)_{f},(\rho c)_{p}$ are the effective heat capacities of the base fluid and nanoparticles. $D_{B}$ and $D_{T}$ denote the Brownian diffusion coefficient and thermophoretic diffusion, respectively. The signs of the coefficients $D_{B}$ and $D_{T}$ are positive and they are respectively equal:

$$
D_{B}=\frac{k_{B} T}{3 \pi \mu d_{p}}, \quad D_{T}=\left(\frac{\mu_{f}}{\rho_{f}}\right)\left(\frac{0.26 k_{f}}{2 k_{f}+k_{p}}\right) \phi,
$$

where $d_{p}$ is the diameter of nanoparticles, $k_{B}$ is the Boltzmann's constant, $k_{f}, k_{p}$ are the thermal conductivity of base nanofluid and nanoparticle, $\mu_{f}$ is the viscosity of base nanofluid.

The external small-scale force $\vec{F}_{0}$ is included in the Navier-Stokes equation (2). This force simulates the excitation source of small-scale and high-frequency pulsations of the velocity field $\vec{v}_{0}$ in the medium with a small Reynolds number $R=\frac{v_{0} t_{0}}{\lambda_{0}} \ll 1$. The main role of the force $\vec{F}_{0}$ is to maintain a moderate level of small-scale movements in the presence of dissipation. An explicit form of the external force $\vec{F}_{0}$ is given below. Let us pass in equations (2)-(5) and boundary conditions (1), (6) to dimensionless variables, which we denoted by the asterisk $(*)$ :

$$
\begin{gathered}
\left(x^{*}, y^{*}, z^{*}\right)=\frac{(x, y, z)}{h}, \vec{V}=\left(V_{x}^{*}, V_{y}^{*}, V_{z}^{*}\right)=\frac{\left(V_{x}, V_{y}, V_{z}\right) h}{\chi_{f}}, t^{*}=\frac{t \cdot \chi_{f}}{h^{2}}, P^{*}=\frac{P \cdot h^{2}}{\chi_{f} \mu}, \\
\vec{\Omega}^{*}=\frac{\vec{\Omega}}{\Omega_{0}}, \varphi^{*}=\frac{\varphi-\varphi_{d}}{\varphi_{u}-\varphi_{d}}, T^{*}=\frac{T-T_{u}}{T_{d}-T_{u}}, \vec{F}_{0}^{*}=\vec{F}_{0} \frac{h^{3}}{\chi_{f} \mu}, \chi_{f}=\frac{k_{f}}{(\rho c)_{f}} .
\end{gathered}
$$

Omitting the asterisk (*) in the system of dimensionless equations (2)-(5) and boundary conditions (1), (6) we get

$$
\begin{gathered}
\frac{1}{\operatorname{Pr}}\left(\frac{\partial \vec{V}}{\partial t}+\vec{V} \cdot \nabla \vec{V}\right)=-\nabla P+\nabla^{2} \vec{V}-\vec{e} R_{n} \varphi-\vec{e} R_{m}+\vec{e} R a T+\sqrt{T a}(\vec{V} \times \vec{\Omega})+\vec{F}_{0} \\
\frac{\partial T}{\partial t}+\vec{V} \cdot \nabla T=\nabla^{2} T+\frac{N_{B}}{L_{e}}(\nabla \varphi \cdot \nabla T)+\frac{N_{A} N_{B}}{L_{e}}(\nabla T \cdot \nabla T) \\
\frac{\partial \varphi}{\partial t}+\vec{V} \cdot \nabla \varphi=\frac{1}{L_{e}} \nabla^{2} \varphi+\frac{N_{A}}{L_{e}} \nabla^{2} T \\
\nabla \cdot \vec{V}=0 \\
T_{d}=1, \varphi_{d}=0, V_{z}=\frac{\partial^{2} V_{z}}{\partial z^{2}}=0 \quad \text { at } \quad z=0 \\
T_{u}=0, \varphi_{u}=1, V_{z}=\frac{\partial^{2} V_{z}}{\partial z^{2}}=0 \quad \text { at } \quad z=1,
\end{gathered}
$$

where $\operatorname{Pr}=\frac{\mu}{\rho_{00} \chi_{f}}$ is the Prandtl number, $R_{n}=\frac{\left(\rho_{p}-\rho_{f}\right)\left(\varphi_{u}-\varphi_{d}\right) g h^{3}}{\mu \chi_{f}}$ is the concentration Rayleigh number, $R_{m}=\frac{\left(\rho_{p} \varphi_{d}+\rho_{f}\left(1-\varphi_{d}\right)\right) g h^{3}}{\mu \chi_{f}}$ is the basic density Rayleigh number, $R a=\frac{\left(T_{d}-T_{u}\right) \rho_{00} g \beta h^{3}}{\mu \chi_{f}}$ is the thermal Rayleigh 
number, $T a=\frac{4 \Omega_{0}^{2} h^{4} \rho_{00}^{2}}{\mu^{2}}$ is the Taylor number, $L_{e}=\chi_{f} / D_{B}$ is the Lewis number, $N_{B}=\left(\varphi_{u}-\varphi_{d}\right) \cdot \frac{(\rho c)_{p}}{(\rho c)_{f}}$ is the modified particle density increment, $N_{A}=\frac{D_{T}\left(T_{d}-T_{u}\right)}{D_{B} T_{u}\left(\varphi_{u}-\varphi_{d}\right)}$ is the modified diffusivity ratio.

We represented all quantities in equations (7)-(10) as the sum of the ground (stationary) and perturbed states:

$$
\vec{V}=\vec{V}^{\prime}, T=T_{b}(z)+T^{\prime}, \varphi=\varphi_{b}(z)+\varphi^{\prime}, P=P_{b}(z)+P^{\prime} .
$$

After substituting (12) into equations (7)-(10), we find the evolution equations for perturbed quantities $\vec{V}^{\prime}, T^{\prime}, \varphi^{\prime}$

$$
\begin{gathered}
\frac{1}{\operatorname{Pr}}\left(\frac{\partial \vec{V}^{\prime}}{\partial t}+\vec{V}^{\prime} \cdot \nabla \vec{V}^{\prime}\right)=-\nabla P^{\prime}+\nabla^{2} \vec{V}^{\prime}-\vec{e} R_{n} \varphi^{\prime}+\vec{e} R a T^{\prime}+\sqrt{T a}\left(\vec{V}^{\prime} \times \vec{\Omega}\right)+\vec{F}_{0} \\
\frac{\partial T^{\prime}}{\partial t}+\vec{V}^{\prime} \cdot \nabla T^{\prime}+V_{z}^{\prime} \frac{d T_{b}}{d z}=\nabla^{2} T^{\prime}+\frac{N_{B}}{L_{e}}\left(\nabla \varphi^{\prime} \cdot \nabla T^{\prime}\right)+\frac{N_{B}}{L_{e}}\left(\frac{d \varphi_{b}}{d z} \frac{d T^{\prime}}{d z}+\frac{d \varphi^{\prime}}{d z} \frac{d T_{b}}{d z}\right)+ \\
+\frac{N_{A} N_{B}}{L_{e}}\left(\nabla T^{\prime} \cdot \nabla T^{\prime}\right)+\frac{2 N_{A} N_{B}}{L_{e}} \frac{d T^{\prime}}{d z} \frac{d T_{b}}{d z} \\
\frac{\partial \varphi^{\prime}}{\partial t}+\vec{V}^{\prime} \cdot \nabla \varphi^{\prime}+V_{z}^{\prime} \frac{d \varphi_{b}}{d z}=\frac{1}{L_{e}} \nabla^{2} \varphi^{\prime}+\frac{N_{A}}{L_{e}} \nabla^{2} T^{\prime}
\end{gathered}
$$

against the background of the ground equilibrium state given by constant gradients of temperature and volume fraction of nanoparticles:

$$
\begin{gathered}
0=-\frac{d P_{b}}{d z}-R_{m}-R_{n} \varphi_{b}+R a T_{b} \\
0=\frac{d^{2} T_{b}}{d z^{2}}+\frac{N_{B}}{L_{e}}\left(\frac{d \varphi_{b}}{d z} \frac{d T_{b}}{d z}\right)+\frac{N_{A} N_{B}}{L_{e}}\left(\frac{d T_{b}}{d z}\right)^{2} \\
0=\frac{d^{2} \varphi_{b}}{d z^{2}}+N_{A} \frac{d^{2} T_{b}}{d z^{2}} .
\end{gathered}
$$

Using the boundary conditions (11), from the equations (14) we find solutions for $T_{b}=1-z$ and $\varphi_{b}=z$, which have a linear dependence on $z$.

Let the external force $\vec{F}_{0}$ have the following properties:

$$
\operatorname{div} \vec{F}_{0}=0, \vec{F}_{0} \operatorname{rot} \vec{F}_{0}=0, \operatorname{rot} \vec{F}_{0} \neq 0, \vec{F}_{0}=f_{0} \vec{F}_{0}\left(\frac{\vec{x}}{\lambda_{0}} ; \frac{t}{t_{0}}\right)
$$

where $\lambda_{0}$ is the characteristic scale, $t_{0}$ is the characteristic time, and $f_{0}$ is the characteristic amplitude. The external force $\vec{F}_{0}$ is specified in the $(X, Y)$ plane orthogonal to the rotation axis and satisfy all properties (15), i.e.

$$
F_{0 z}=0, \vec{F}_{0 \perp}=f_{0}\left(\vec{i} \cos \phi_{2}+\vec{j} \cos \phi_{1}\right), \phi_{1}=k_{0} x-\omega_{0} t, \phi_{2}=k_{0} y-\omega_{0} t
$$

Here $\omega_{0}, k_{0}$ are the frequency and wavenumber of the external parametric action, respectively. A simple physical form of an external force (16) can be easily implemented in laboratory experiments. Let us rescale the variables in the equations for perturbations (13):

$$
\begin{gathered}
\vec{x} \rightarrow \frac{\vec{x}}{\lambda_{0}}, t \rightarrow \frac{t}{t_{0}}, \vec{V} \rightarrow \frac{\vec{V}^{\prime}}{v_{0}}, P \rightarrow \frac{P^{\prime}}{p_{0}}, \vec{F}_{0} \rightarrow \frac{\vec{F}_{0}}{f_{0}}, \\
T \rightarrow \frac{T^{\prime}}{R}, \varphi \rightarrow \frac{\varphi^{\prime}}{R}, \frac{p_{0} t_{0}}{\lambda_{0} v_{0}}=\frac{f_{0} t_{0}}{v_{0}}=\frac{t_{0}}{\lambda_{0}^{2}}=1 .
\end{gathered}
$$

As a result, we obtained the following system of equations for perturbations 


$$
\begin{gathered}
\frac{1}{\operatorname{Pr}}\left(\frac{\partial \vec{V}}{\partial t}+R \vec{V} \cdot \nabla \vec{V}\right)=-\nabla P+\nabla^{2} \vec{V}-\vec{e} \widetilde{R}_{n} \varphi+\vec{e} \widetilde{R a} T+\vec{V} \times \vec{D}+\vec{F}_{0} \\
\frac{\partial T}{\partial t}+R \vec{V} \cdot \nabla T-V_{z}=\nabla^{2} T+R \frac{N_{B}}{L_{e}}(\nabla \varphi \cdot \nabla T)+\frac{N_{B}}{L_{e}}\left(\frac{d T}{d z}-\frac{d \varphi}{d z}\right)+ \\
+R \frac{N_{A} N_{B}}{L_{e}}(\nabla T \cdot \nabla T)-\frac{2 N_{A} N_{B}}{L_{e}} \frac{d T}{d z} \\
\frac{\partial \varphi}{\partial t}+R \vec{V} \cdot \nabla \varphi+V_{z}=\frac{1}{L_{e}} \nabla^{2} \varphi+\frac{N_{A}}{L_{e}} \nabla^{2} T
\end{gathered}
$$

where the new notation is introduced

$$
\vec{D}=\frac{2 h^{2}}{\mu} \rho_{00} \lambda_{0}^{2} \vec{\Omega}, \quad \widetilde{R}_{n}=R_{n} \cdot \lambda_{0}^{3}, \quad \widetilde{R a}=R a \cdot \lambda_{0}^{3} .
$$

Let us consider the Reynolds number $R$ for small-scale motions to be a small parameter of the asymptotic expansion and assume $\vec{D}, \widetilde{R}_{n}, \widetilde{R a}$ to be arbitrary parameters that do not affect the expansion scheme. We consider the external force as being of small scale and high frequency. This force leads to small scale fluctuations in velocity. After averaging, these rapidly oscillating fluctuations vanish. Nevertheless, due to small nonlinear interactions in some orders of perturbation theory, nonzero terms can occur after averaging. In the next section, we consider in detail how to find the solvability conditions for the multi-scale asymptotic expansion, which define the evolution equations for large-scale perturbations.

\section{EQUATIONS FOR LARGE-SCALE FIELDS}

In this section, we consider in more detail the application of the method of multiscale asymptotic expansions to the problem of nonlinear evolution of large-scale vortex disturbances in an obliquely rotating nanofluid. The method of asymptotic equations is well presented in works [6-7]. Following these papers we introduce spatial and temporal derivatives in equations (17)-(19) in the form of asymptotic expansions:

$$
\frac{\partial}{\partial t} \rightarrow \partial_{t}+R^{4} \partial_{T}, \quad \frac{\partial}{\partial x_{i}} \rightarrow \partial_{i}+R^{2} \nabla_{i}
$$

where $\partial_{i}$ and $\partial_{t}$ denote derivatives concerning fast variables $x_{0}=\left(\vec{x}_{0}, t_{0}\right)$ and $\nabla_{i}, \partial_{T}$ derivatives concerning slow variable $X=(\vec{X}, T)$. Variables $x_{0}$ and $X$ can be called small-scale and large-scale variables. To construct the nonlinear theory, the variables $\vec{V}, T, \varphi, P$ are presented in the form of asymptotic series:

$$
\begin{gathered}
\vec{V}(\vec{x}, t)=\frac{1}{R} \vec{W}_{-1}(X)+\vec{v}_{0}+R \vec{v}_{1}+R^{2} \vec{v}_{2}+R^{3} \vec{v}_{3}+\cdots \\
T(\vec{x}, t)=\frac{1}{R} T_{-1}(X)+T_{0}+R T_{1}+R^{2} T_{2}+R^{3} T_{3}+\cdots \\
\varphi(\vec{x}, t)=\frac{1}{R} \varphi_{-1}(X)+\varphi_{0}+R \varphi_{1}+R^{2} \varphi_{2}+R^{3} \varphi_{3}+\cdots \\
P(x)=\frac{1}{R^{3}} P_{-3}+\frac{1}{R^{2}} P_{-2}+\frac{1}{R} P_{-1}+P_{0}+R\left(P_{1}+\bar{P}_{1}(X)\right)+R^{2} P_{2}+R^{3} P_{3}+\cdots
\end{gathered}
$$

By substituting developments (20)-(21) into the initial equations (17)-(19) and then gathering together the terms of the same order, we obtained the equations of the multi-scale asymptotic development and wrote down the obtained equations up to order $R^{3}$ including. The algebraic structure of the asymptotic development of equations (17)-(19) in various orders of $R$ is given in Appendix A. It is also shown that in the order $R^{3}$ we got the main secular equation or equation for the large-scale fields in a stratified nanofluid: 


$$
\begin{gathered}
\frac{1}{\operatorname{Pr}}\left(\partial_{T} W_{-1}^{i}+\nabla_{k}\left(\overline{v_{0}^{k} v_{0}^{i}}\right)\right)=-\nabla_{i} \bar{P}_{1}+\Delta W_{-1}^{i} \\
\partial_{T} T_{-1}+\nabla_{k}\left(\overline{v_{0}^{k} T_{0}}\right)=\Delta T_{-1}+\frac{N_{B}}{L_{e}}\left(\nabla_{k} \varphi_{-1} \nabla_{k} T_{-1}\right)+\frac{N_{A} N_{B}}{L_{e}}\left(\nabla_{k} T_{-1} \nabla_{k} T_{-1}\right) \\
\partial_{T} \varphi_{-1}+\nabla_{k}\left(\overline{v_{0}^{k} \varphi_{0}}\right)=\frac{1}{L_{e}} \Delta \varphi_{-1}+\frac{N_{A}}{L_{e}} \Delta T_{-1}
\end{gathered}
$$

Equations (22)-(24) are supplemented by the secular equations obtained in Appendix A:

$$
\begin{gathered}
-\nabla_{i} P_{-3}-e_{i} \widetilde{R}_{n} \varphi_{-1}+e_{i} \widetilde{R a} T_{-1}+\varepsilon_{i j k} W_{j} D_{k}=0, \\
W_{-1}^{z}=0, \quad \nabla_{i} W_{-1}^{i}=0, \\
\frac{1}{\operatorname{Pr}} W_{-1}^{k} \nabla_{k} W_{-1}^{i}=-\nabla_{i} P_{-1}, \\
W_{-1}^{k} \nabla_{k} T_{-1}=\frac{N_{B}}{L_{e}}\left(\partial_{Z} T_{-1}-\partial_{Z} \varphi_{-1}\right)-\frac{2 N_{A} N_{B}}{L_{e}} \partial_{Z} T_{-1}, \\
W_{-1}^{k} \nabla_{k} \varphi_{-1}=0 .
\end{gathered}
$$

The influence of small-scale oscillations excited by the external force $\vec{F}_{0}$ on the evolution of large-scale motion $\vec{W}_{-1}$ is described by the equation (22). It can be seen from this equation that the large-scale temperature $T_{-1}$ and the volume fraction of nanoparticles $\varphi_{-1}$ do not influence on the dynamics of the large-scale velocity field $\vec{W}_{-1}$. Therefore, we restrict ourselves to studying the equation (22). This equation takes on a closed-form after calculating the correlation function - Reynolds stress $\nabla_{k} \overline{\left(v_{0}^{k} v_{0}^{i}\right)}$. The calculation of the Reynolds stress will be greatly simplified if we use the «quasi-two-dimensional» approximation, where the horizontal scales significantly exceed the vertical ones. As a part of this approach, the large-scale derivatives according to are more preferable, i.e

$$
\nabla_{Z} \equiv \frac{\partial}{\partial Z} \gg \frac{\partial}{\partial X}, \frac{\partial}{\partial Y}
$$

and the geometry of large-scale fields is following:

$$
\vec{W}_{-1}=\left(W_{-1}^{x}(Z), W_{-1}^{y}(Z), 0\right), T_{-1}=T_{-1}(Z), \varphi_{-1}=\varphi_{-1}(Z), P_{-1}=\text { const }
$$

Then the equation (22) is simplified and takes the following form:

$$
\begin{aligned}
& \partial_{T} W_{1}-\nabla_{Z}^{2} W_{1}+\nabla_{Z}\left(\overline{v_{0}^{z} v_{0}^{x}}\right)=0, \quad W_{-1}^{x}=W_{1} \\
& \partial_{T} W_{2}-\nabla_{Z}^{2} W_{2}+\nabla_{Z}\left(\overline{v_{0}^{z} v_{0}^{y}}\right)=0, \quad W_{-1}^{y}=W_{2}
\end{aligned}
$$

Equations (31)-(32) describe the evolution of large-scale eddy fields $\vec{W}$. In order to obtain the final closed form of equations (31)-(32) we have to calculate the Reynolds stresses $\nabla_{k}\left(\overline{v_{0}^{k} v_{0}^{i}}\right)$. This shows that we need to find solutions for the small-scale velocity field $\vec{v}_{0}$. Appendix B contains a detailed technique to calculate the velocity field in a rotating stratified nanofluid. Further, in Appendix C solutions for small-scale velocity field $\vec{v}_{0}$ are used to find the Reynolds stresses. Then equations (31)-(32) take a closed form:

$$
\left(\partial_{T}-\nabla_{Z}^{2}\right) W_{1}=-\nabla_{Z}\left(\frac{f_{0}^{2} D_{2} k_{0}^{2}\left(k_{0}^{4}+\widetilde{\omega}_{2}^{2}-\widetilde{R a}-l_{n_{2}}\right)}{2\left(k_{0}^{4}+\widetilde{\omega}_{2}^{2}\right) \Lambda_{2}}\right)
$$




$$
\left(\partial_{T}-\nabla_{Z}^{2}\right) W_{2}=\nabla_{Z}\left(\frac{f_{0}^{2} D_{1} k_{0}^{2}\left(k_{0}^{4}+\widetilde{\omega}_{1}^{2}-\widetilde{R a}-l_{n_{1}}\right)}{2\left(k_{0}^{4}+\widetilde{\omega}_{1}^{2}\right) \Lambda_{1}}\right)
$$

We used here the following notations:

$$
\begin{aligned}
& \tilde{\omega}_{1,2}^{2}=\left(\omega_{0}-k_{0} W_{1,2}\right)^{2}, \quad \Lambda_{1,2}= \\
& \left(k_{0}^{4}+\operatorname{Pr}^{-2} \widetilde{\omega}_{1,2}^{2}\right)\left(k_{0}^{4}+\operatorname{Pr}^{-2} \widetilde{\omega}_{1,2}^{2}+\frac{\widetilde{R a}^{2}}{k_{0}^{4}+\widetilde{\omega}_{1,2}^{2}}-2 \widetilde{R a} \cdot \frac{k_{0}^{4}-\operatorname{Pr}^{-1} \widetilde{\omega}_{1,2}^{2}}{k_{0}^{4}+\widetilde{\omega}_{1,2}^{2}}+r_{n_{1,2}}\right)+ \\
& +2 D_{1,2}^{2}\left(k_{0}^{4}-\operatorname{Pr}^{-2} \widetilde{\omega}_{1,2}^{2}-\widetilde{R a} \cdot \frac{k_{0}^{4}+\operatorname{Pr}^{-1} \widetilde{\omega}_{1,2}^{2}}{k_{0}^{4}+\widetilde{\omega}_{1,2}^{2}}+p_{n_{1,2}}\right)+D_{1,2}^{4}, \\
& r_{n_{1,2}}=-2 \widetilde{R}_{n} \cdot \frac{\left(k_{0}^{4}+\widetilde{\omega}_{1,2}^{2}\right)\left(L_{e}^{-1} k_{0}^{4}-\operatorname{Pr}^{-1} \widetilde{\omega}_{1,2}^{2}\right)+\frac{N_{A}}{L_{e}} k_{0}^{2}\left(L_{e}^{-1} k_{0}^{2}-\operatorname{Pr}^{-1} \widetilde{\omega}_{1,2}^{2} k_{0}^{2}-\widetilde{\omega}_{1,2}^{2} k_{0}^{2}\left(1+\operatorname{Pr}^{-1} L_{e}^{-1}\right)\right)}{\left(k_{0}^{4}+\widetilde{\omega}_{1,2}^{2}\right)\left(L_{e}^{-2} k_{0}^{4}+\widetilde{\omega}_{1,2}^{2}\right)}+ \\
& +\widetilde{R}_{n}^{2} \cdot \frac{k_{0}^{4}\left(1+\frac{2 N_{A}}{L_{e}}+\frac{N_{A}^{2}}{L_{e}^{2}}\right)+\widetilde{\omega}_{1,2}^{2}}{\left(k_{0}^{4}+\widetilde{\omega}_{1,2}^{2}\right)\left(L_{e}^{-2} k_{0}^{4}+\widetilde{\omega}_{1,2}^{2}\right)}+2 \widetilde{R}_{n} \widetilde{R a} \cdot \frac{\widetilde{\omega}_{1,2}^{2}+k_{0}^{2} L_{e}^{-1}\left(1+\frac{N_{A}}{L_{e}}\right)}{\left(k_{0}^{4}+\widetilde{\omega}_{1,2}^{2}\right)\left(L_{e}^{-2} k_{0}^{4}+\widetilde{\omega}_{1,2}^{2}\right)} \\
& p_{n_{1,2}}=-\widetilde{R}_{n} \cdot \frac{\left(k_{0}^{4}+\widetilde{\omega}_{1,2}^{2}\right)\left(L_{e}^{-1} k_{0}^{4}+\operatorname{Pr}^{-1} \widetilde{\omega}_{1,2}^{2}\right)+\frac{N_{A}}{L_{e}} k_{0}^{4}\left(L_{e}^{-1} k_{0}^{4}+\operatorname{Pr}^{-1} \widetilde{\omega}_{1,2}^{2}+\widetilde{\omega}_{1,2}^{2}\left(\operatorname{Pr}^{-1} L_{e}^{-1}-1\right)\right)}{\left(k_{0}^{4}+\widetilde{\omega}_{1,2}^{2}\right)\left(L_{e}^{-2} k_{0}^{4}+\widetilde{\omega}_{1,2}^{2}\right)}, \\
& l_{n_{1,2}}=\widetilde{R}_{n} \cdot \frac{L_{e}^{-1}+\frac{N_{A}}{L_{e}}\left(k_{0}^{2} L_{e}^{-1}-\frac{\widetilde{\omega}_{1,2}^{2}}{k_{0}^{2}}\right)}{L_{e}^{-2} k_{0}^{4}+\widetilde{\omega}_{1,2}^{2}} \cdot\left(k_{0}^{4}+\widetilde{\omega}_{1,2}^{2}\right) .
\end{aligned}
$$

Thus, in this section, we obtained the closed equations (33)-(34), which are called the equations of nonlinear vortex dynamo in obliquely rotating stratified nanofluid under the parametric action of a small-scale non-spiral force. The large-scale fields decay due to viscous dissipation if rotation $(\Omega=0)$ or external force $\left(f_{0}=0\right)$ disappears. For the limit of a homogeneous fluid $\widetilde{R a}=\widetilde{R}_{n}=0$ and the absence of nanoparticles, the equation (33)-(34) coincide with the results of [8]. At first, we will consider the stability of small perturbations of fields (linear theory).

\section{LARGE-SCALE INSTABILITY}

Equations (33)-(34) describe the nonlinear dynamics of large scale disturbances of the vortex field $\vec{W}=\left(W_{1}, W_{2}\right)$. Therefore it is interesting to clarify the question of the stability of small perturbations of the field $\vec{W}$. Then for small values $\left(W_{1}, W_{2}\right)$ the equations (33)-(34) are linearized and can be reduced to the following system of linear equations:

$$
\left\{\begin{array}{l}
\partial_{T} W_{1}-\nabla_{Z}^{2} W_{1}-\alpha_{2} \nabla_{Z} W_{2}=0 \\
\partial_{T} W_{2}-\nabla_{Z}^{2} W_{2}+\alpha_{1} \nabla_{Z} W_{1}=0
\end{array}\right.
$$

here $\alpha_{1.2}$ are the coefficients of the linear vortex dynamo:

$$
\alpha_{1.2}=\frac{f_{0}^{2}}{2} \frac{D_{1,2} k_{0}^{2}}{\Lambda_{0(1,2)}}\left(R+\frac{\Lambda_{(1,2)}}{\Lambda_{0(1,2)}}\left(R_{0}-1\right)\right), R_{0}=\frac{\widetilde{R a}}{k_{0}^{4}+\omega_{0}^{2}}+\frac{a_{6} \widetilde{R}_{n}}{\widetilde{a}_{0}\left(k_{0}^{4}+\omega_{0}^{2}\right)}
$$




$$
\begin{gathered}
R=\frac{2 k_{0} \omega_{0} \widetilde{R a}}{\left(k_{0}^{4}+\omega_{0}^{2}\right)^{2}}+\frac{\widetilde{R}_{n}}{\tilde{a}_{0}\left(k_{0}^{4}+\omega_{0}^{2}\right)} \cdot\left(b_{6}+\frac{a_{6} \tilde{b}_{0}}{\tilde{a}_{0}}+\frac{2 k_{0} \omega_{0} a_{6}}{k_{0}^{4}+\omega_{0}^{2}}\right), \\
\Lambda_{0(1,2)}=\left(k_{0}^{4}+\operatorname{Pr}^{-2} \omega_{0}^{2}\right)^{2}+2\left(D_{1,2}^{2}-\xi_{0} \widetilde{R a}\right)\left(k_{0}^{4}-\operatorname{Pr}^{-2} \omega_{0}^{2}\right)+\xi_{0}\left(D_{1,2}^{2}-\widetilde{R a}\right)^{2}+\xi_{0}^{\prime} D_{1,2}^{4}+ \\
+\frac{a_{4}}{a_{0}}\left(k_{0}^{4}+\operatorname{Pr}^{-2} \omega_{0}^{2}\right)-2 D_{1,2}^{2} \widetilde{R}_{n} \frac{a_{5}}{a_{0}}, \\
\Lambda_{1,2}=4 k_{0} \omega_{0} \operatorname{Pr}^{-2}\left(k_{0}^{4}+\operatorname{Pr}^{-2} \omega_{0}^{2}\right)-2\left[2 k_{0} \omega_{0} \operatorname{Pr}^{-2}\left(D_{1,2}^{2}-\xi_{0} \widetilde{R a}\right)-\xi \xi_{1} \widetilde{R a}\left(k_{0}^{4}-\operatorname{Pr}^{-2} \omega_{0}^{2}\right)\right]- \\
-\xi_{1}\left(D_{1,2}^{2}-\widetilde{R a}\right)^{2}-\xi_{1} D_{1,2}^{4}-\frac{a_{4} b_{0}-b_{4} a_{0}}{a_{0}^{2}}\left(k_{0}^{4}+\operatorname{Pr}^{-2} \omega_{0}^{2}\right)+2 k_{0} \omega_{0} \operatorname{Pr}^{-2} \frac{a_{4}}{a_{0}}+2 D_{1,2}^{2} \widetilde{R}_{n} \cdot \frac{a_{5} b_{0}-b_{5} a_{0}}{a_{0}^{2}} .
\end{gathered}
$$

Here the coefficients $a_{6}, b_{6}, \tilde{a}_{0}, \tilde{b}_{0}, \xi_{0}, \xi_{1}, \xi_{0}^{\prime}, \xi_{1}^{\prime}, a_{5}, b_{5}, a_{4}, b_{4}, a_{3}, b_{3}, a_{2}, b_{2} . a_{1}, b_{1}, a_{0}, b_{0}$ included in (36) have the form:

$$
\begin{gathered}
a_{6}=\left(k_{0}^{4}+\omega_{0}^{2}\right)\left(L_{e}^{-1}+\frac{N_{A}}{L_{e}}\left(k_{0}^{2} L_{e}^{-1}-\frac{\omega_{0}^{2}}{k_{0}^{2}}\right)\right), \\
b_{6}=\frac{2 N_{A} \omega_{0}}{L_{e} k_{0}}\left(k_{0}^{4}+\omega_{0}^{2}\right)-2 k_{0} \omega_{0}\left(L_{e}^{-1}+\frac{N_{A}}{L_{e}}\left(\frac{k_{0}^{2}}{L_{e}}-\frac{\omega_{0}^{2}}{k_{0}^{2}}\right)\right), \\
\tilde{a}_{0}=L_{e}^{-2} k_{0}^{4}+\omega_{0}^{2}, \tilde{b}_{0}=2 k_{0} \omega_{0}, \xi_{0}=\frac{k_{0}^{4}+\operatorname{Pr}^{-2} \omega_{0}^{2}}{k_{0}^{4}+\omega_{0}^{2}}, \xi_{1}=\frac{2 k_{0}^{5} \omega_{0}\left(1-\operatorname{Pr}^{-2}\right)}{\left(k_{0}^{4}+\omega_{0}^{2}\right)^{2}}, \\
\xi_{0}^{\prime}=\frac{\omega_{0}^{2}\left(1-\operatorname{Pr}^{-2}\right)}{k_{0}^{4}+\omega_{0}^{2}}, \xi_{1}^{1}=\frac{2 k_{0}^{5} \omega_{0}\left(1-\operatorname{Pr}^{-2}\right)}{\left(k_{0}^{4}+\omega_{0}^{2}\right)^{2}}, a_{4}=-2 \widetilde{R}_{n} a_{1}+\widetilde{R}_{n}^{2} a_{2}+2 a_{3} \widetilde{R}_{n} \widetilde{R a}, \\
b_{4}=2 \widetilde{R}_{n} b_{1}+\widetilde{R}_{n}^{2} b_{2}+2 b_{3} \widetilde{R}_{n} \widetilde{R a}, a_{1}=\left(k_{0}^{4}+\omega_{0}^{2}\right)\left(L_{e}^{-1} k_{0}^{4}-\operatorname{Pr}^{-1} \omega_{0}^{2}\right)+ \\
k_{0}^{2}\left(L_{e} k_{0}^{2}-\omega_{0}^{2} k_{0}^{2}\left(1+\operatorname{Pr}^{-1}+\operatorname{Pr}^{-1} L_{e}^{-1}\right)\right), b_{1}=2 k_{0} \omega_{0}\left(\operatorname{Pr}^{-1}\left(k_{0}^{4}+\omega_{0}^{2}\right)-L_{e}^{-1} k_{0}^{4}+\omega_{0}^{2} \operatorname{Pr}^{-1}\right)+ \\
+2 k_{0}^{5} \omega_{0}\left(1+\operatorname{Pr}^{-1}+\operatorname{Pr}^{-1} L_{e}^{-1}\right) \frac{N_{A}}{L_{e}}, a_{2}=k_{0}^{4}\left(1+\frac{2 N_{A}}{L_{e}}+\frac{N_{A}^{2}}{L_{e}^{2}}\right)+\omega_{0}^{2}, b_{2}=2 k_{0} \omega_{0}, \\
b_{5}=2 k_{0} \omega_{0}\left[k_{0}^{4}\left(L_{e}^{-1}+\operatorname{Pr}_{e}^{-1}\right)+2 \omega_{0}^{2} \operatorname{Pr}^{-1}+\frac{N_{A}}{L_{e}} k_{0}^{4}\left(\operatorname{Pr}^{-1}\left(1+L_{e}^{-1}\right)-1\right)\right] . \\
a_{3}=\omega_{0}^{2}+L_{e}^{-1} k_{0}^{2}\left(1+\frac{N_{A}}{L_{e}}\right), b_{3}=b_{2}, a_{0}=\left(k_{0}^{4}+\omega_{0}^{2}\right)\left(L_{e}^{-2} k_{0}^{4}+\omega_{0}^{2}\right), \\
b_{0}=2 k_{0} \omega_{0}\left(k_{0}^{4}\left(1+L_{e}^{-2}\right)+2 \omega_{0}^{2}\right), a_{5}=\left(k_{0}^{4}+\omega_{0}^{2}\right)\left(k_{0}^{4} L_{e}^{-1}+\omega_{0}^{2} \operatorname{Pr}^{-1}\right)+
\end{gathered}
$$

We choose perturbations $\left(W_{1}, W_{2}\right)$ in (35) in the form of plane waves with the wave vector $\vec{K} \| O Z$, i. e.

$$
W_{1}=A_{W_{1}} \exp (\Gamma t) \sin K Z, \quad W_{2}=A_{W_{2}} \exp (\Gamma t) \cos K Z
$$

The solutions (37) describe a circularly polarized plane wave whose amplitude increases exponentially with time. Such 
waves are called Beltrami waves because the large-scale velocity field (37) satisfies the Beltrami flow under which the following condition is fulfilled:

$$
\vec{W} \times \operatorname{rot} \vec{W}=0
$$

Substituting (37) into the system of equations (35) we obtained the dispersion equation:

$$
\left(\Gamma+K^{2}\right)^{2}-\alpha_{1} \alpha_{2} K^{2}=0
$$

From here we found:

$$
\Gamma= \pm \sqrt{\alpha_{1} \alpha_{2}} K-K^{2}
$$

Solutions (39) show the existence of instabilities for large-scale vortical perturbations when $\alpha_{1} \alpha_{2}>0$. When $\alpha_{1} \alpha_{2}<0$, damped oscillations arise with frequency $\omega_{0}=\sqrt{\alpha_{1} \alpha_{2}} K$ instead of the instabilities. The maximum increment of instability $\Gamma_{\max }=\frac{\alpha_{1} \alpha_{2}}{4}$ is reached on the wavenumber $K_{\max }=\frac{\sqrt{\alpha_{1} \alpha_{2}}}{2}$. Coefficients $\alpha_{1}, \alpha_{2}$ give a positive feedback loop between velocity components. It should be noted that in the linear theory, the coefficients $\alpha_{1}, \alpha_{2}$ do not depend on field amplitudes but depend only on dimensionless parameters $D_{1,2}, \widetilde{R a}, \operatorname{Pr}, \widetilde{R}_{n}, N_{A}, L_{e}$ and the external force amplitude $f_{0}$. Let us analyze the dependence of these coefficients on the dimensionless parameters assuming, for simplicity, that the dimensionless amplitude of the external force $f_{0}$ is equal to $f_{0}=10$. Fixing the level of dimensionless force means the selection of a certain level of the stationary background of small-scale and fast oscillations. As for the coefficients $\alpha_{1}, \alpha_{2}$, instead of the Cartesian projections $D_{1}$ and $D_{2}$ it is convenient to use their projections in the spherical coordinate system $(D, \varphi, \theta)$ (see Fig. 2). The coordinate surface $D=$ const is the sphere, $\theta$ is the latitude $\theta \in[0, \pi], \varphi$ is the longitude $\varphi \in[0,2 \pi]$. Let us analyze the dependence of the coefficients $\alpha_{1}, \alpha_{2}$ on the effects of rotation, stratification and concentration of nanoparticles. Then we assumed $D_{1}=D_{2}$ for simplicity that corresponds to a fixed value of longitude $\varphi=\pi / 4+\pi n$, where $n=0,1,2 \ldots k, k$ is an integer. In this case, the amplification coefficients of the vortex perturbations are, respectively, equal to:

$$
\alpha_{n f}=\alpha_{1}=\alpha_{2}=\frac{f_{0}^{2}}{4} \sqrt{2} D \sin \theta \frac{k_{0}^{2}}{\Lambda_{0}(\theta)}\left(R+\frac{\Lambda(\theta)}{\Lambda_{0}(\theta)}\left(R_{0}-1\right)\right)
$$

It should be noted that at inclination angles $(\theta=0, \theta=\pi)$ vortex disturbances are not generated since $\alpha_{n f}=0$. It is also interesting to find out how the coefficient $\alpha_{n f}$ depends on the inclination angle $\theta$ of the nanofluid rotation axis.

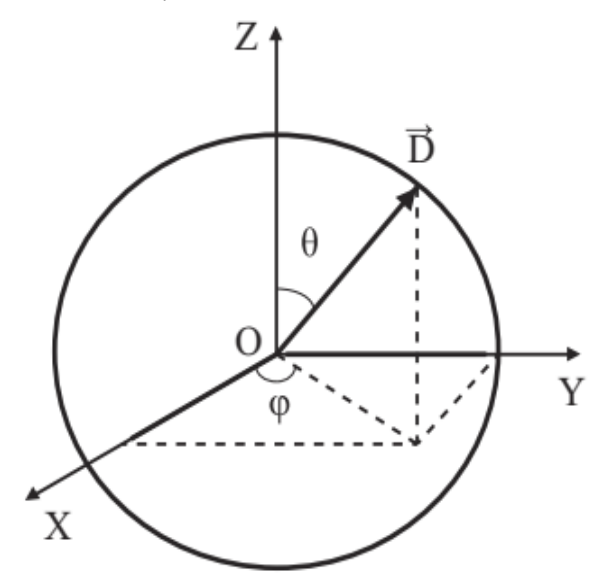

Figure 2. Relation of Cartesian projections of rotation parameter $\vec{D}$ (or angular rotation velocity $\vec{\Omega}$ ) with their projections in a spherical coordinate system.

This dependence $\alpha_{n f}(\theta)$ is shown in Fig. 3 by a solid line obtained for fixed values $D=2, \widetilde{R a}=2, k_{0}=\omega_{0}=1, \operatorname{Pr}=5, \widetilde{R}_{n}=0.122, N_{A}=5, L_{e}=5000$. The values of the nanofluid parameters $\operatorname{Pr}, \widetilde{R}_{n}, N_{A}, L_{e}$ ( $\mathrm{Al}_{2} \mathrm{O}_{3}$-water) are taken from [2]. As seen from Fig. 3 a maximum value of $\alpha_{n f}$ for nanofluid is inclination angle $\theta_{\max } \approx \pi / 5+\pi n$, and a minimum value for $\theta_{\min } \approx \pi / 2+\pi n$. The dashed line in Fig. 3 a corresponds to the dependence 
$\alpha_{b}(\theta)$ on the case of a pure fluid with the Prandtl number $\operatorname{Pr}=5$. Graphs Fig. 3a shows that the maximum coefficient $\alpha_{b}=\left(\alpha_{n f}\right)_{\tilde{R}_{n}=0}$ for a pure fluid is greater than for a nanofluid. This conclusion remains valid for the Prandtl numbers $\operatorname{Pr}=1$. In this case, the maximum coefficients $\alpha_{n f}^{(b)}$ in nano- and pure fluid are at inclination angles $\theta \approx \pi / 2+\pi n$ (see Fig. 3b).

a)

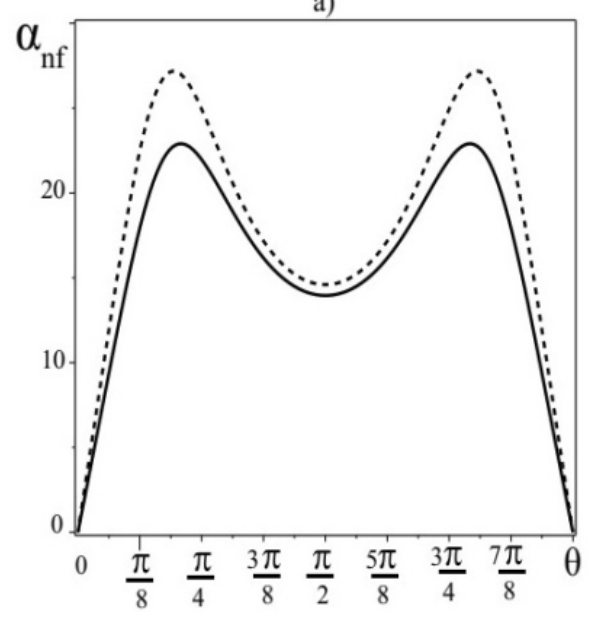

b)

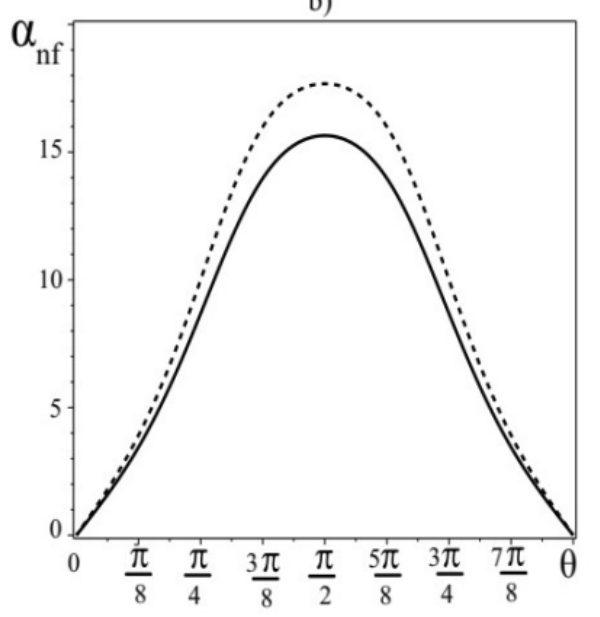

Figure 3. The solid line shows the dependence of the coefficient $\alpha_{n f}$ for nanofluid on the inclination angle $\theta$ and the dashed line shows the dependence of the coefficient $\alpha_{b}$ for the pure liquid on the inclination angle $\theta$. Graphs a) and b) are plotted for the Prandtl numbers $\operatorname{Pr}=5$ and $\operatorname{Pr}=1$ respectively.

Consequently, the characteristic time $T_{n f}$ and the characteristic scale $L_{n f}$ of generated large-scale vortices in nanofluid exceed the corresponding scales $T_{b}, L_{b}$ in a pure fluid

$$
T_{n f} \gg T_{b}, \quad L_{n f} \gg L_{b}, T_{n f} \approx\left(\alpha_{n f}^{2} / 4\right)^{-1}, L_{n f} \approx\left(\alpha_{n f} / 2\right)^{-1}, T_{b} \approx\left(\alpha_{b}^{2} / 4\right)^{-1}, L_{b} \approx\left(\alpha_{b} / 2\right)^{-1}
$$

Let us consider the influence of the nanofluid rotation effect on the coefficient $\alpha_{n f}$ or the process of generation of large-scale vortices. For this, we fixed the parameters of the nanofluid $\operatorname{Pr}, \widetilde{R}_{n}, N_{A}, L_{e}$ and the Rayleigh number $\widetilde{R a}=2$. We chose the inclination angle equal to $\theta_{\max } \simeq \pi / 5$, which corresponds to maximum values of the coefficient $\alpha_{n f}$ (see Fig. 3a). As see from Fig. 4a for a certain value of the rotation parameter $D$ the coefficient $\alpha_{n f}\left(\alpha_{b}\right)$ reaches its maximum value $\alpha_{\max }^{(n f, b)}$. Then with the increasing of $D$ the coefficient $\alpha$ tends gradually to zero i. e. the suppression of $\alpha$ - effect occurs. A similar phenomenon was described in [9].
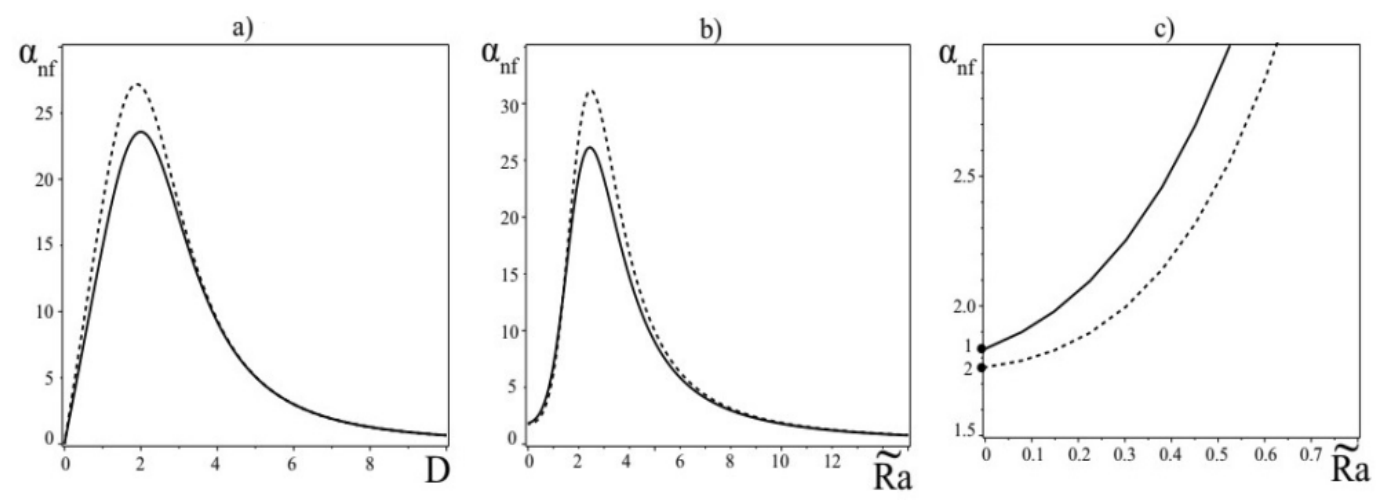

Figure 4. a) The solid line shows the dependence of the coefficient $\alpha_{n f}$ for the nanofluid on the rotation parameter $D$, and the dashed line shows the dependence of the coefficient $\alpha_{b}$ for the pure liquid on the rotation parameter $D$; b) The solid line shows the dependence of the coefficient $\alpha_{n f}$ for the nanofluid on the Rayleigh number $\widetilde{R a}$, and the dashed line shows the dependence of the coefficient $\alpha_{b}$ for the pure fluid on the Rayleigh number $\widetilde{R a}$; c) point 1 corresponds to the value of $\alpha_{n f}$ (nanofluid) at $\widetilde{R a}=0$, point 2 corresponds to the value of $\alpha_{b}$ (pure fluid) at $\widetilde{R a}=0$. 
Next, fixing the parameters of nanofluid $\operatorname{Pr}, \widetilde{R}_{n}, N_{A}, L_{e}$, rotation $D=2$ and inclination angle $\theta_{\max } \simeq \pi / 5$, we determine the dependence of the coefficient $\alpha_{n f}$ of Rayleigh number $\widetilde{R a}$. From the graphs Fig. $4 \mathrm{~b}$ it can be seen that the maximum value of the coefficient $\alpha_{n f}\left(\alpha_{b}\right)$ corresponds to small Rayleigh numbers $\widetilde{R a}$. Large-scale vortices are efficiently generated in the range of Rayleigh numbers $\widetilde{R a} \in[0,3]$, then we observed the decay $\alpha_{n f}\left(\alpha_{b}\right)$ (Fig. 4b) with an increase in $\widetilde{R a}$ and the generation of the LSVS becomes ineffective. It means that for large Rayleigh numbers $\widetilde{R a}$, large-scale instability in nano- and ordinary fluids is not realized and the ordinary convective instability is arisen. Under the condition that there is no heating $\widetilde{R a}=0$, the gain coefficient in pure fluid $\alpha_{b}$ (point 2 in Fig. 4c) is less than in nanofluid $\alpha_{n f}$ (point 1 in Fig. 4c): $\alpha_{n f}>\alpha_{b}$. In Fig. 4a-4b it is also seen that the maximum coefficient $\alpha_{b}=\left(\alpha_{n f}\right)_{\tilde{R}_{n}=0}$ is larger for pure fluid than for nanofluid.

In Fig. 5a is a graph showing the combined effects of rotation and temperature stratification in the plane $(D, \widetilde{R a})$. Here the instability region $\alpha_{n f}>0$ is highlighted in gray. Curve 1 corresponds to the instability boundary for nanofluid $\left(\widetilde{R}_{n}=0.122\right)$ and curve 2 corresponds to the instability boundary for pure fluid $\left(\widetilde{R}_{n}=0\right)$.

Let us analyze the influence of the Rayleigh concentration number $\widetilde{R}_{n}$ on the coefficient $\alpha_{n f}$ or the generation of LSVS for the following fixed parameters $D=2, \widetilde{R a}=3, k_{0}=\omega_{0}=1, \operatorname{Pr}=5, N_{A}=5, L_{e}=5000, \theta \simeq \pi / 5$. In Fig. $5 \mathrm{~b}$ shows the intersection of graphs (curve 1 and curve 2) at the point $C\left(\widetilde{R}_{n}^{(0)}, \alpha_{n f}^{(0)}\right)$. Curve 1 is plotted for the case when there is a temperature gradient $\widetilde{R a}=3$. For $\widetilde{R}_{n}=0$, curve 1 shows the maximum value of $\alpha_{b}^{\text {st }}$ (point $A$ ) corresponding to a pure stratified fluid.

a)

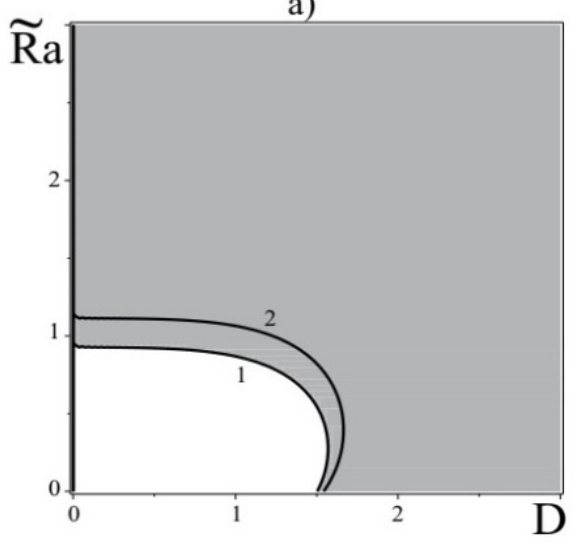

b)

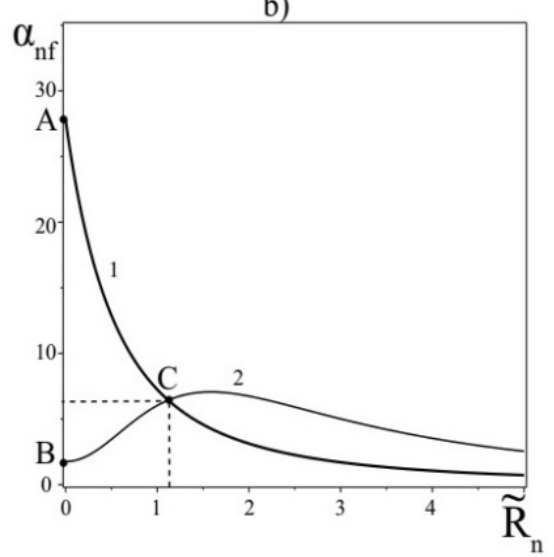

Figure 5. a) The plot for $\alpha_{n f}$ in the plane $(D, \widetilde{R a})$, where the gray color shows the region corresponding to positive values $\alpha$ (unstable solutions), and the white color region shows negative values $\alpha$. Curve 1 corresponds to the instability boundary for nanofluid ( $\widetilde{R}_{n}=0.122$ ), and curve 2 corresponds to the instability boundary for pure fluid $\left(\widetilde{R}_{n}=0\right)$. b) The plot of the dependence on the $\alpha_{n f} 9$ - effect on the Rayleigh concentration number $\widetilde{R}_{n}$.

A further increase in the concentration of nanoparticles leads to a decrease in $\alpha_{n f}$. Curve 2 is plotted for the case when there is no temperature gradient $\widetilde{R a}=0$. It can be seen from the behavior of curve 2 that an increase in the concentration of nanoparticles at first leads to an increase in $\alpha_{n f}$, and then to a decrease. For $\widetilde{R}_{n}=0$, curve 2 shows the maximum value of $\alpha_{b}^{h}$ (point $B$ ), corresponding to the coefficient $\alpha$ for a homogeneous fluid [8]. Here we see that in a pure stratified fluid the generation of LSVS is more efficient than in a homogeneous fluid, which is consistent with the conclusions of the work [6]. Thus, for a certain value of the number $\widetilde{R}_{n}^{(0)}$ (concentration of nanoparticles), we obtained equal rates generation of LSVS (point $C$ in Fig. 5 b) in nanofluid as in the presence of a temperature gradient $\widetilde{R a} \neq 0$, and without it $\widetilde{R a}=0$. Physically, this process can be explained as follows. An increase in the concentration of nanoparticles on the upper surface layer leads to the appearance of a flow due to the gravitational segregation of nanoparticles to the lower surface. In turn, the presence of a temperature gradient arises a heat flux $\vec{q} \sim \vec{e}\left(T_{d}-T_{u}\right) / h$, which prevents the deposition of nanoparticles on the lower surface layer. An increase in the concentration of nanoparticles $\widetilde{R}_{n}$ decreases the part of the heat flux and as a consequence, the coefficient $\alpha_{n f}$ is decreased. 
a)

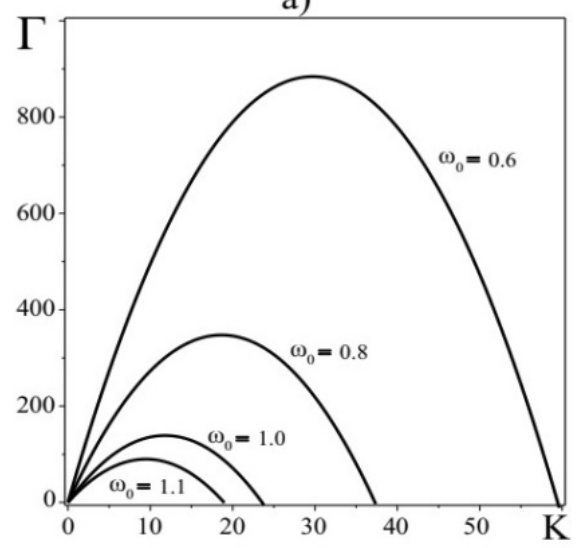

b)

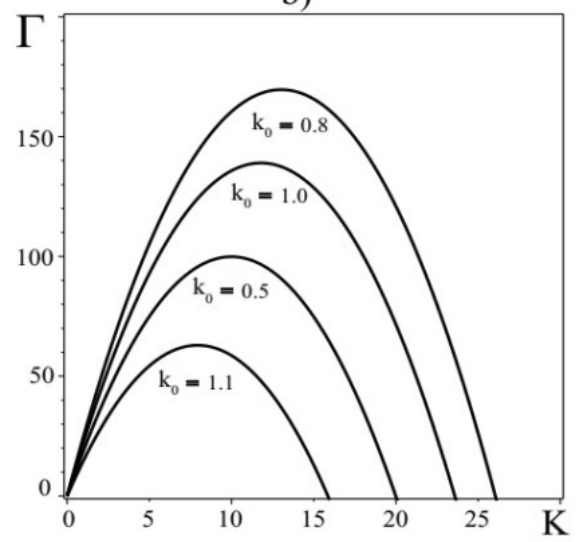

Figure 6. a) The graph of the dependence on the instability growth rate $\Gamma$ on wavenumbers $K$ for different frequencies $\omega_{0}$ of the external force $\vec{F}_{0}$ at $k_{0}=1$. b) The graph of the dependence on the instability growth rate $\Gamma$ on wavenumbers $K$ for different wavenumbers $k_{0}$ of the external force $\vec{F}_{0}$ at $\omega_{0}=1$.

Fig. 6 shows the dependence on the instability increment $\Gamma$ on the wavenumbers $K$ for the hydrodynamic $\alpha_{n f}$ effect in nanofluid at constant parameters $D=2, \widetilde{R a}=2, \operatorname{Pr}=5, \widetilde{R}_{n}=0.122, N_{A}=5, L_{e}=5000, \theta \simeq \pi / 5$. As it is shown in Fig. 6a with an increase in the frequency $\omega_{0}$ of the external force $\vec{F}_{0}$ at $k_{0}=1$ the maximum growth rate $\Gamma_{\max }$ of large-scale vortex disturbances is decreased. Fixing the frequency of the external force $\vec{F}_{0}$ at $\omega_{0}=1$ we constructed a graph of the increment $\Gamma(K)$ (see Fig. 6b) when the small-scale wavenumber $k_{0}$ changes. For numbers $k_{0}<1$ we observed an increase in the maximum growth rate $\Gamma_{\max }$ of large-scale vortex disturbances $\left(k_{0}=0.8\right)$ relative to the level $\Gamma_{\max }$ at $k_{0}=1$, and a decrease the maximum growth rate of large-scale vortex disturbances at $k_{0}=0.5$. This behavior is due to the structural dependence of the coefficient $\alpha_{n f}$ on the small-scale parameters of the external force $\left(\omega_{0}, k_{0}\right)$.

Thus, as a result of the large-scale instability development in an obliquely rotating stratified nanofluid, the largescale spiral circularly polarized Beltrami-type vortices are generated.

\section{INSTABILITY SATURATION AND NON LINEAR STRUCTURES}

The increase of $W_{1}$ and $W_{2}$ leads to the saturation of the instability. As a result of the development and stabilization of the instability, non-linear structures appear. The study of these structures is of interest. In order to find these structures let us examine the stationary case of equations (33)-(34) and integrate once by $Z$. For the sake of simplicity, we assume that $D_{1}=D_{2}, \theta=\pi / 2$ (latitude), $\phi=\pi / 4$ (longitude), and Prandtl number $\operatorname{Pr}=1$. As a result, we get a system of nonlinear equations of the following form:

$$
\begin{gathered}
\frac{d W_{1}}{d Z}=\frac{f_{0}^{2} \sqrt{2}}{4} \frac{D k_{0}^{2}\left(k_{0}^{4}+\widetilde{\omega}_{2}^{2}-\widetilde{R a}-l_{n_{2}}\right)}{2\left(k_{0}^{4}+\widetilde{\omega}_{2}^{2}\right) \Lambda_{2}}+C_{1}, \\
\frac{d W_{2}}{d Z}=-\frac{f_{0}^{2} \sqrt{2}}{4} \frac{D k_{0}^{2}\left(k_{0}^{4}+\widetilde{\omega}_{1}^{2}-\widetilde{R a}-l_{n_{1}}\right)}{2\left(k_{0}^{4}+\widetilde{\omega}_{2}^{2}\right) \Lambda_{1}}+C_{2} .
\end{gathered}
$$

Here $C_{1}, C_{2}$ are arbitrary constants of integration. It should be noted that the dynamic system of equations (40)-(41) is conservative, and hence is Hamiltonian. It's easy to find it we write down the equations (40)-(41) in the Hamiltonian form:

$$
\frac{\mathrm{d} W_{1}}{\mathrm{~d} Z}=\frac{\mathrm{d} H}{\mathrm{~d} W_{2}}, \quad \frac{\mathrm{d} W_{2}}{\mathrm{~d} Z}=-\frac{\mathrm{d} H}{\mathrm{~d} W_{1}},
$$

where the Hamiltonian has the form:

$$
H=H_{1}\left(W_{1}\right)+H_{2}\left(W_{2}\right)+C_{1} W_{2}-C_{2} W_{1}
$$


The functions $H_{1,2}$ are respectively equal to:

$$
H_{1,2}=\frac{f_{0}^{2} \sqrt{2}}{4} D \sin \theta \int \frac{k_{0}^{2}\left(k_{0}^{4}+\widetilde{\omega}_{1,2}^{2}-\widetilde{R a}-l_{n_{1,2}}\right) \mathrm{d} W_{1,2}}{2 \operatorname{Pr}\left(k_{0}^{4}+\widetilde{\omega}_{2}^{2}\right) \Lambda_{1,2}}
$$

The integral in the Hamiltonian $H_{1,2}$ cannot be calculated exactly in quadratures. We used the values of the nanofluid parameters $\operatorname{Pr}=1, \widetilde{R}_{n}=0.122, N_{A}=5, L_{e}=5000$ (for $\mathrm{Al}_{2} \mathrm{O}_{3}$-water) from the paper [2]. The equations (40)-(41) are Hamiltonian thus only fixed points of two types: elliptic and hyperbolic can be observed in phase space. This can be checked if we carry out a qualitative analysis of the system of equations (40)-(41). Linearizing the right-hand sides of equations (40)-(41) in the neighborhood of fixed points, we establish their type and construct a phase portrait. As a result of the analysis, we find the appearance of four fixed points, two of hyperbolic and two of elliptic type. Phase portrait of a dynamical system of equations (40)-(41) for the constants $C_{1}=-0.005, C_{2}=0.005$ and parameters $D=\widetilde{R a}=2, k_{0}=\omega_{0}=1, f_{0}=10$ is shown in Fig. 7.

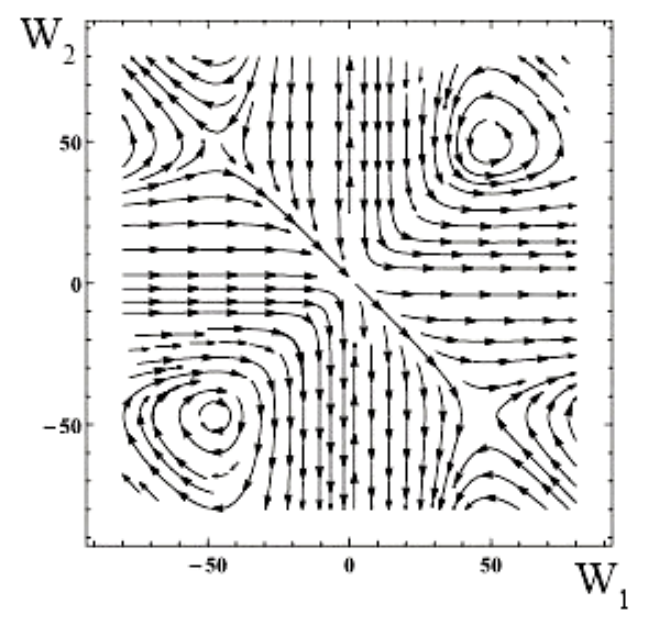

Figure 7. The phase plane of the dynamical system equations (40)-(41) with $C_{1}=-0.05$ and $C_{2}=0.05$. One can see the presence of closed trajectories around the elliptic points and separatrices that connect the hyperbolic points.

The phase portrait allows us to describe qualitatively the possible stationary solutions. The most interesting localized solutions correspond to the phase portrait trajectories, which connect the stationary (singular) points on the phase plane. Fig. 7 presents closed trajectories on the phase plane around the elliptic points and separatrices which connect the hyperbolic points. Closed trajectories correspond to nonlinear periodic solutions or nonlinear waves. The separatrices correspond to localized vortex structures of the kinks type (see Fig. 8).
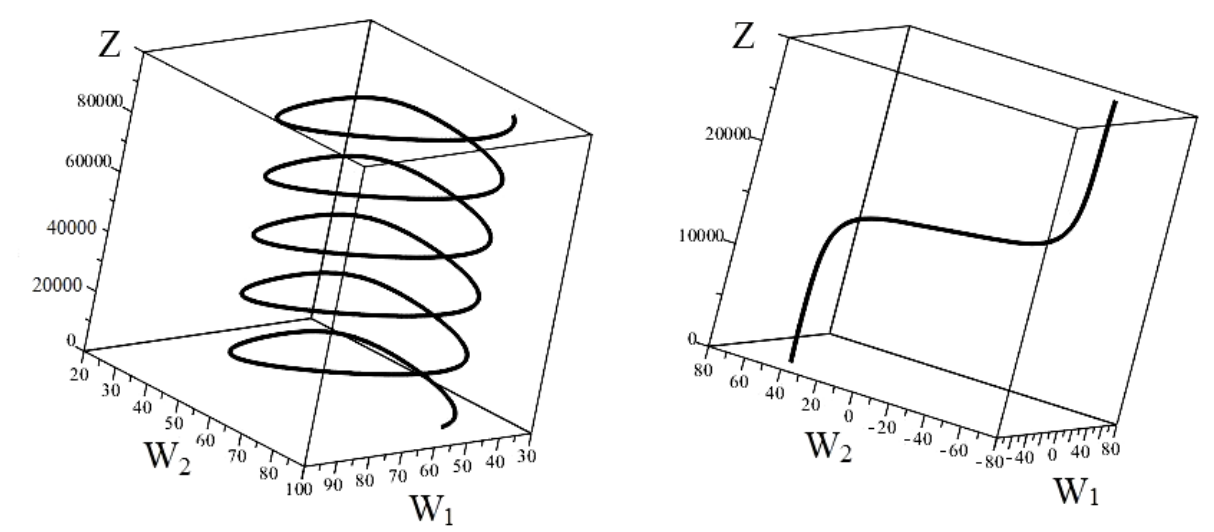

Figure 8. On the left a nonlinear helical wave, which corresponds to a closed trajectory on the phase plane; on the right a localized nonlinear vortex structure (kink), which corresponds to the separatrix on the phase plane.

\section{CONCLUSION}

In this work, we have obtained a new type of large-scale instability caused by the temperature gradient and the specific concentration of nanoparticles gradient under the action of a small-scale force with zero helicity $\vec{F}_{0} r o t \vec{F}_{0}=0$ in 
an obliquely rotating nanofluid. This force maintains small-scale fluctuations in the nanofluid and simulates the action of small-scale turbulence with Reynolds number $R \ll 1$. We assumed that the external force is in the plane $(X, Y)$ and the gravity field is directed $\vec{g}$ vertically downward along an axis $O Z$. Applying the method of multiscale asymptotic expansions we obtained a closed system of equations for large-scale perturbations of the nanofluid velocity $\vec{W}$. For small amplitudes $\vec{W}$ this system of equations describes the instability of the hydrodynamic $\alpha$-effect, since positive feedback appears between the velocity components. We have shown that the instability occurs only when the vector of the angular velocity of rotation is inclined from the axis $O Z$. Moreover, for the Prandtl numbers $\mathrm{Pr}=5$, the maximum instability increment reaches inclination angles $\theta \approx \pi / 5$, and for the Prandtl numbers $\operatorname{Pr}=1$ inclination angles $\theta \approx \pi / 2$. Taking into account the effects of rotation and temperature stratification of the nanofluid together leads to a significant increase in large-scale vortex disturbances in contrast to the case of a homogeneous medium [8]. In addition, we have obtained a new effect of generation of LSVS in nanofluids at $\widetilde{R a}=0$ associated with an increase in the concentration of nanoparticles (see Fig. 5b, curve 2). With an increase in the frequency of the parametric action of an external force, the generation of LSVS becomes less efficient. This effect allows you to control and monitor the process of generating LSVS. The instability becomes nonlinear with increasing amplitude $\vec{W}$. In this case, the emergence of stationary nonlinear vortex structures is assumed. The numerical solutions show that in the non-linear stage, the instability saturation leads to specific velocity profiles (kinks) for which the velocity tends to be constant for large values of $Z$. The phase portrait contains elliptic stationary points therefore, there are nonlinear periodic solutions in the form of nonlinear Beltrami waves that correspond to closed trajectories. These structures are the result of saturation of large-scale instability (see section LARGE-SCALE INSTABILITY). Obtained results can find their applications in many problems of laboratory experiments on rotating stratified nanofluids.

\section{A. MULTISCALE ASYMPTOTIC DEVELOPMENTS}

APPENDIX

Let us find the algebraic structure of the asymptotic development in various orders of $R$, starting from the lowest one. In order of $R^{-3}$ there is only one equation:

$$
\partial_{i} P_{-3}=0 \Rightarrow P_{-3}=P_{-3}(X)
$$

In order $R^{-2}$ appears the equations:

$$
\partial_{i} P_{-2}=0 \Rightarrow P_{-2}=P_{-2}(X)
$$

In order $R^{-1}$, we obtain the more complicated system of equations:

$$
\begin{gathered}
\frac{1}{\operatorname{Pr}}\left(\partial_{t} W_{-1}^{i}+W_{-1}^{k} \partial_{k} W_{-1}^{i}\right)=-\partial_{i} P_{-1}-\nabla_{i} P_{-3}+\partial_{k}^{2} W_{-1}^{i}-e_{i} \widetilde{R}_{n} \varphi_{-1}+e_{i} \widetilde{R a} T_{-1}+\varepsilon_{i j k} W_{j} D_{k} \\
\partial_{t} T_{-1}+W_{-1}^{k} \partial_{k} T_{-1}-W_{-1}^{z}=\partial_{k}^{2} T_{-1}+\frac{N_{B}}{L_{e}} \partial_{k} \varphi_{-1} \partial_{k} T_{-1}+\frac{N_{B}}{L_{e}}\left(\partial_{z} T_{-1}-\partial_{z} \varphi_{-1}\right)+ \\
+\frac{N_{A} N_{B}}{L_{e}}\left(\partial_{k} T_{-1} \partial_{k} T_{-1}\right)-\frac{2 N_{A} N_{B}}{L_{e}} \partial_{z} T_{-1} \\
\partial_{t} \varphi_{-1}+W_{-1}^{k} \partial_{k} \varphi_{-1}+W_{-1}^{z}=\frac{1}{L_{e}} \partial_{k}^{2} \varphi_{-1}+\frac{N_{A}}{L_{e}} \partial_{k}^{2} T_{-1} \\
\partial_{i} W_{-1}^{i}=0
\end{gathered}
$$

The averaging of equations (46)-(49) over the fast variables gives the following secular equations:

$$
\begin{gathered}
-\nabla_{i} P_{-3}-e_{i} \widetilde{R}_{n} \varphi_{-1}+e_{i} \widetilde{R a} T_{-1}+\varepsilon_{i j k} W_{j} D_{k}=0 \\
W_{-1}^{z}=0
\end{gathered}
$$

In zero order in $R^{0}$ we have the equations:

$$
\frac{1}{\operatorname{Pr}}\left(\partial_{t} v_{0}^{i}+W_{-1}^{k} \partial_{k} v_{0}^{i}+v_{0}^{k} \partial_{k} W_{-1}^{i}\right)=-\partial_{i} P_{0}-\nabla_{i} P_{-2}+\partial_{k}^{2} v_{0}^{i}-e_{i} \widetilde{R}_{n} \varphi_{0}+e_{i} \widetilde{R} a T_{0}+\varepsilon_{i j k} v_{0}^{j} D_{k}+F_{0}^{i}
$$




$$
\begin{gathered}
\partial_{t} T_{0}+W_{-1}^{k} \partial_{k} T_{0}+v_{0}^{k} \partial_{k} T_{-1}-v_{0}^{z}=\partial_{k}^{2} T_{0}+\frac{N_{B}}{L_{e}}\left(\partial_{k} \varphi_{-1} \partial_{k} T_{0}+\partial_{k} \varphi_{0} \partial_{k} T_{-1}\right)+ \\
+\frac{N_{B}}{L_{e}}\left(\partial_{z} T_{0}-\partial_{z} \varphi_{0}\right)+\frac{N_{A} N_{B}}{L_{e}}\left(\partial_{k} T_{-1} \partial_{k} T_{0}+\partial_{k} T_{0} \partial_{k} T_{-1}\right)-\frac{2 N_{A} N_{B}}{L_{e}} \partial_{z} T_{0} \\
\partial_{t} \varphi_{0}+W_{-1}^{k} \partial_{k} \varphi_{0}+v_{0}^{k} \partial_{k} \varphi_{-1}+v_{0}^{z}=\frac{1}{L_{e}} \partial_{k}^{2} \varphi_{0}+\frac{N_{A}}{L_{e}} \partial_{k}^{2} T_{0} \\
\partial_{i} v_{0}^{i}=0
\end{gathered}
$$

These equations give one secular equation:

$$
\nabla P_{-2}=0 \Rightarrow P_{-2}=\text { const }
$$

Let us consider the equations of the first approximation $R^{1}$ :

$$
\begin{gathered}
\frac{1}{\operatorname{Pr}}\left(\partial_{t} v_{1}^{i}+W_{-1}^{k} \partial_{k} v_{1}^{i}+v_{0}^{k} \partial_{k} v_{0}^{i}+v_{1}^{k} \partial_{k} W_{-1}^{i}+W_{-1}^{k} \nabla_{k} W_{-1}^{i}\right)=-\nabla_{i} P_{-1}-\partial_{i}\left(P_{1}+\bar{P}_{1}\right)+\partial_{k}^{2} v_{1}^{i}+ \\
+2 \partial_{k} \nabla_{k} W_{-1}^{i}-e_{i} \widetilde{R}_{n} \varphi_{1}+e_{i} \widetilde{R a} T_{1}+\varepsilon_{i j k} v_{1}^{j} D_{k} \\
\partial_{t} T_{1}+W_{-1}^{k} \partial_{k} T_{1}+W_{-1}^{k} \nabla_{k} T_{-1}+v_{0}^{k} \partial_{k} T_{0}+v_{1}^{k} \partial_{k} T_{-1}-v_{1}^{z}=\partial_{k}^{2} T_{1}+2 \partial_{k} \nabla_{k} T_{-1}+ \\
+\frac{N_{B}}{L_{e}}\left(\partial_{k} \varphi_{-1} \partial_{k} T_{1}+\partial_{k} \varphi_{-1} \nabla_{k} T_{-1}+\partial_{k} \varphi_{0} \partial_{k} T_{0}+\partial_{k} \varphi_{1} \partial_{k} T_{-1}+\nabla_{k} \varphi_{-1} \partial_{k} T_{-1}\right)+ \\
+\frac{N_{A} N_{B}}{L_{e}}\left(\partial_{k} T_{-1} \partial_{k} T_{1}+\partial_{k} T_{-1} \nabla_{k} T_{-1}+\partial_{k} T_{0} \partial_{k} T_{0}+\partial_{k} T_{1} \partial_{k} T_{-1}+\nabla_{k} T_{-1} \partial_{k} T_{-1}\right)+ \\
+\frac{N_{B}}{L_{e}}\left(\partial_{z} T_{1}+\partial_{Z} T_{-1}-\partial_{z} \varphi_{1}-\partial_{Z} \varphi_{-1}\right)-\frac{2 N_{A} N_{B}}{L_{e}}\left(\partial_{z} T_{1}+\partial_{Z} T_{-1}\right) \\
\partial_{t} \varphi_{1}+W_{-1}^{k} \partial_{k} \varphi_{1}+W_{-1}^{k} \nabla_{k} \varphi_{-1}+v_{0}^{k} \partial_{k} \varphi_{0}+v_{1}^{k} \partial_{k} \varphi_{-1}+v_{1}^{z}= \\
=\frac{1}{L_{e}}\left(\partial_{k}^{2} \varphi_{1}+2 \partial_{k} \nabla_{k} \varphi_{-1}\right)+\frac{N_{A}}{L_{e}}\left(\partial_{k}^{2} T_{1}+2 \partial_{k} \nabla_{k} T_{-1}\right) \\
\partial_{i} v_{1}^{i}+\nabla_{i} W_{-1}^{i}=0
\end{gathered}
$$

The secular equations follow from this system of equations:

$$
\begin{gathered}
\frac{1}{\operatorname{Pr}} W_{-1}^{k} \nabla_{k} W_{-1}^{i}=-\nabla_{i} P_{-1}, \quad W_{-1}^{k} \nabla_{k} T_{-1}=\frac{N_{B}}{L_{e}}\left(\partial_{Z} T_{-1}-\partial_{Z} \varphi_{-1}\right)-\frac{2 N_{A} N_{B}}{L_{e}} \partial_{Z} T_{-1}, \\
W_{-1}^{k} \nabla_{k} \varphi_{-1}=0, \quad \nabla_{i} W_{-1}^{i}=0
\end{gathered}
$$

Secular equations (61) satisfy the following field geometry:

$$
\vec{W}_{-1}=\left(W_{-1}^{x}(Z), W_{-1}^{y}(Z), 0\right), T_{-1}=T_{-1}(Z), \varphi_{-1}=\varphi_{-1}(Z), P_{-1}=\text { const }
$$

In the second order $R^{2}$, we obtain the equations:

$$
\frac{1}{\operatorname{Pr}}\left(\partial_{t} v_{2}^{i}+W_{-1}^{k} \partial_{k} v_{2}^{i}+v_{0}^{k} \partial_{k} v_{1}^{i}+W_{-1}^{k} \nabla_{k} v_{0}^{i}+v_{0}^{k} \nabla_{k} W_{-1}^{i}+v_{1}^{k} \partial_{k} v_{0}^{i}+v_{2}^{k} \partial_{k} W_{-1}^{i}\right)=
$$




$$
\begin{gathered}
=-\partial_{i} P_{2}-\nabla_{i} P_{0}+\partial_{k}^{2} v_{2}^{i}+2 \partial_{k} \nabla_{k} v_{0}^{i}-e_{i} \widetilde{R}_{n} \varphi_{2}+e_{i} \widetilde{R a} T_{2}+\varepsilon_{i j k} v_{2}^{j} D_{k} \\
\partial_{t} T_{2}+W_{-1}^{k} \partial_{k} T_{2}+W_{-1}^{k} \nabla_{k} T_{0}+v_{0}^{k} \partial_{k} T_{1}+v_{0}^{k} \nabla_{k} T_{-1}+v_{1}^{k} \partial_{k} T_{0}+v_{2}^{k} \partial_{k} T_{-1}-v_{2}^{z}= \\
=\partial_{k}^{2} T_{2}+2 \partial_{k} \nabla_{k} T_{0}+ \\
+\frac{N_{B}}{L_{e}}\left(\partial_{k} \varphi_{-1} \partial_{k} T_{2}+\partial_{k} \varphi_{-1} \nabla_{k} T_{0}+\partial_{k} \varphi_{0} \partial_{k} T_{1}+\partial_{k} \varphi_{0} \nabla_{k} T_{-1}+\partial_{k} \varphi_{1} \partial_{k} T_{0}+\right. \\
\left.+\partial_{k} \varphi_{2} \partial_{k} T_{-1}+\nabla_{k} \varphi_{-1} \partial_{k} T_{0}+\nabla_{k} \varphi_{0} \partial_{k} T_{-1}\right)+ \\
+\frac{N_{A} N_{B}}{L_{e}}\left(\partial_{k} T_{-1} \partial_{k} T_{2}+\partial_{k} T_{-1} \nabla_{k} T_{0}+\partial_{k} T_{0} \partial_{k} T_{1}+\partial_{k} T_{0} \nabla_{k} T_{-1}+\partial_{k} T_{1} \partial_{k} T_{0}+\right. \\
\left.+\partial_{k} T_{2} \partial_{k} T_{-1}+\nabla_{k} T_{-1} \partial_{k} T_{0}+\nabla_{k} T_{0} \partial_{k} T_{-1}\right)+ \\
+\frac{N_{B}}{L_{e}}\left(\partial_{z} T_{2}+\partial_{Z} T_{0}-\partial_{z} \varphi_{2}-\partial_{z} \varphi_{0}\right)-\frac{2 N_{A} N_{B}}{L_{e}}\left(\partial_{z} T_{2}+\partial_{z} T_{0}\right) \\
\partial_{t} \varphi_{2}+W_{-1}^{k} \partial_{k} \varphi_{2}+W_{-1}^{k} \nabla_{k} \varphi_{0}+v_{0}^{k} \partial_{k} \varphi_{1}+v_{0}^{k} \nabla_{k} \varphi_{-1}+v_{1}^{k} \partial_{k} \varphi_{0}+v_{2}^{k} \partial_{k} \varphi_{-1}+v_{2}^{z}= \\
=\frac{1}{L_{e}}\left(\partial_{k}^{2} \varphi_{2}+2 \partial_{k} \nabla_{k} \varphi_{0}\right)+\frac{N_{A}}{L_{e}}\left(\partial_{k}^{2} T_{2}+2 \partial_{k} \nabla_{k} T_{0}\right) \\
\partial_{i} v_{2}^{i}+\nabla_{i} v_{0}^{i}=0
\end{gathered}
$$

After averaging the system of equations (63)-(66) over the fast variables, it can be seen that there are no secular terms in the order $R^{2}$. Finally, we come to the most important order $R^{3}$. In this order, the equations are following

$$
\begin{gathered}
\frac{1}{\operatorname{Pr}}\left(\partial_{t} v_{3}^{i}+\partial_{T} W_{-1}^{i}+W_{-1}^{k} \partial_{k} v_{3}^{i}+v_{0}^{k} \partial_{k} v_{2}^{i}+W_{-1}^{k} \nabla_{k} v_{1}^{i}+v_{0}^{k} \nabla_{k} v_{0}^{i}+v_{1}^{k} \partial_{k} v_{1}^{i}+v_{1}^{k} \nabla_{k} W_{-1}^{i}+\right. \\
\left.+v_{2}^{k} \partial_{k} v_{0}^{i}+v_{3}^{k} \partial_{k} W_{-1}^{i}\right)=-\partial_{i} P_{3}-\nabla_{i}\left(P_{1}+\bar{P}_{1}\right)+\partial_{k}^{2} v_{3}^{i}+2 \partial_{k} \nabla_{k} v_{1}^{i}+\Delta W_{-1}^{i}- \\
-e_{i} \widetilde{R}_{n} \varphi_{3}+e_{i} \widetilde{R a} T_{3}+\varepsilon_{i j k} v_{3}^{j} D_{k} \\
\partial_{t} T_{3}+\partial_{T} T_{-1}+W_{-1}^{k} \partial_{k} T_{3}+W_{-1}^{k} \nabla_{k} T_{1}+v_{0}^{k} \partial_{k} T_{2}+v_{0}^{k} \nabla_{k} T_{0}+v_{1}^{k} \nabla_{k} T_{1}+ \\
+v_{1}^{k} \nabla_{k} T_{-1}+v_{2}^{k} \partial_{k} T_{0}+v_{3}^{k} \partial_{k} T_{-1}-v_{3}^{z}=\partial_{k}^{2} T_{3}+2 \partial_{k} \nabla_{k} T_{1}+\Delta T_{-1}+ \\
+\frac{N_{B}}{L_{e}}\left(\partial_{k} \varphi_{-1} \partial_{k} T_{3}+\partial_{k} \varphi_{-1} \nabla_{k} T_{1}+\partial_{k} \varphi_{0} \partial_{k} T_{2}+\partial_{k} \varphi_{0} \nabla_{k} T_{0}+\partial_{k} \varphi_{1} \partial_{k} T_{1}+\partial_{k} \varphi_{1} \nabla_{k} T_{-1}+\right. \\
\left.+\partial_{k} \varphi_{2} \partial_{k} T_{0}+\partial_{k} \varphi_{3} \partial_{k} T_{-1}+\nabla_{k} \varphi_{-1} \partial_{k} T_{1}+\nabla_{k} \varphi_{-1} \nabla_{k} T_{-1}+\nabla_{k} \varphi_{0} \partial_{k} T_{0}+\nabla_{k} \varphi_{1} \partial_{k} T_{-1}\right)+ \\
+\frac{N_{A} N_{B}}{L_{e}}\left(\partial_{k} T_{-1} \partial_{k} T_{3}+\partial_{k} T_{-1} \nabla_{k} T_{1}+\partial_{k} T_{0} \partial_{k} T_{2}+\partial_{k} T_{0} \nabla_{k} T_{0}+\partial_{k} T_{1} \partial_{k} T_{1}+\partial_{k} T_{1} \nabla_{k} T_{-1}+\right. \\
\left.+\partial_{k} T_{2} \partial_{k} T_{0}+\partial_{k} T_{3} \partial_{k} T_{-1}+\nabla_{k} T_{-1} \partial_{k} T_{1}+\nabla_{k} T_{-1} \nabla_{k} T_{-1}+\nabla_{k} T_{0} \partial_{k} T_{0}+\nabla_{k} T_{1} \partial_{k} T_{-1}\right)+ \\
+\frac{N_{B}}{L_{e}}\left(\partial_{z} T_{3}+\partial_{Z} T_{1}-\partial_{z} \varphi_{3}-\partial_{Z} \varphi_{1}\right)-\frac{2 N_{A} N_{B}}{L_{e}}\left(\partial_{z} T_{3}+\partial_{Z} T_{1}\right)
\end{gathered}
$$




$$
\begin{gathered}
\partial_{t} \varphi_{3}+\partial_{T} \varphi_{-1}+W_{-1}^{k} \partial_{k} \varphi_{3}+W_{-1}^{k} \nabla_{k} \varphi_{1}+v_{0}^{k} \partial_{k} \varphi_{2}+v_{0}^{k} \nabla_{k} \varphi_{0}+v_{1}^{k} \partial_{k} \varphi_{1}+v_{1}^{k} \nabla_{k} \varphi_{-1}+v_{2}^{k} \partial_{k} \varphi_{0}+ \\
+v_{3}^{k} \partial_{k} \varphi_{-1}+v_{3}^{z}=\frac{1}{L_{e}}\left(\partial_{k}^{2} \varphi_{3}+2 \partial_{k} \nabla_{k} \varphi_{1}+\Delta \varphi_{-1}\right)+\frac{N_{A}}{L_{e}}\left(\partial_{k}^{2} T_{3}+2 \partial_{k} \nabla_{k} T_{1}+\Delta T_{-1}\right) \\
\partial_{i} v_{3}^{i}+\nabla_{i} v_{1}^{i}=0
\end{gathered}
$$

Averaging the system of equations (67)-(70) over the fast variables, we will obtain the basic secular equations that describe the evolution of large-scale perturbations:

$$
\begin{gathered}
\frac{1}{\operatorname{Pr}}\left(\partial_{T} W_{-1}^{i}+\nabla_{k}\left(\overline{v_{0}^{k} v_{0}^{i}}\right)\right)=-\nabla_{i} \bar{P}_{1}+\Delta W_{-1}^{i} \\
\partial_{T} T_{-1}+\nabla_{k}\left(\overline{v_{0}^{k} T_{0}}\right)=\Delta T_{-1}+\frac{N_{B}}{L_{e}}\left(\nabla_{k} \varphi_{-1} \nabla_{k} T_{-1}\right)+\frac{N_{A} N_{B}}{L_{e}}\left(\nabla_{k} T_{-1} \nabla_{k} T_{-1}\right) \\
\partial_{T} \varphi_{-1}+\nabla_{k}\left(\overline{v_{0}^{k} \varphi_{0}}\right)=\frac{1}{L_{e}} \Delta \varphi_{-1}+\frac{N_{A}}{L_{e}} \Delta T_{-1}
\end{gathered}
$$

\section{B. SMALL-SCALE FIELDS IN THE ZERO ORDER IN $R$}

Let us consider Eqs. (52)-(55) for the zeroth order in $R$ derived in Appendix A. By introducing the notation for the operators

$$
\begin{gathered}
\widehat{D}_{W}=\operatorname{Pr}^{-1}\left(\partial_{t}+W_{-1}^{k} \partial_{k}\right)-\partial^{2}, \quad \widehat{D}_{T}=\partial_{t}+W_{-1}^{k} \partial_{k}-\partial^{2}-\frac{N_{B}}{L_{e}} \partial_{z}+\frac{2 N_{A} N_{B}}{L_{e}} \partial_{z}, \\
\widehat{D}_{\varphi}=\partial_{t}+W_{-1}^{k} \partial_{k}-L_{e}^{-1} \partial^{2}
\end{gathered}
$$

we can write the system of equations (52)-(55) as

$$
\begin{gathered}
\widehat{D}_{W} v_{0}^{i}=-\partial_{i} P_{0}-e_{i} \widetilde{R}_{n} \varphi_{0}+e_{i} \widetilde{R a} T_{0}+\varepsilon_{i j k} v_{0}^{j} D_{k}+F_{0}^{i} \\
\widehat{D}_{T} T_{0}=v_{0}^{z}-\frac{N_{B}}{L_{e}} \partial_{z} \varphi_{0} \\
\widehat{D}_{\varphi} \varphi_{0}=-v_{0}^{z}+\frac{N_{A}}{L_{e}} \partial^{2} T_{0} \\
\partial_{i} v_{0}^{i}=0
\end{gathered}
$$

Small-scale oscillations of temperature and volume fraction of nanoparticles are easily found from the equations (75)(76):

$$
T_{0}=\frac{\widehat{D}_{\varphi}+\frac{N_{B}}{L_{e}} \partial_{z}}{\widehat{L}} v_{0}^{z}, \quad \varphi_{0}=-\frac{\widehat{D}_{T}-\frac{N_{A}}{L_{e}} \partial^{2}}{\widehat{L}} v_{0}^{z}, \quad \hat{L}=\widehat{D}_{\varphi} \widehat{D}_{T}+\frac{N_{A} N_{B}}{L_{e}^{2}} \partial_{z} \partial^{2}
$$

Let us substitute (78) in (74) and using the condition of solenoidality of the fields $\vec{v}_{0}$ and $\vec{F}_{0}$, we obtain the pressure $P_{0}$ :

$$
P_{0}=\widehat{P}_{1} u_{0}+\widehat{P}_{2} v_{0}+\widehat{P}_{3} w_{0}
$$

Where we introduce the designations for operators

$$
\widehat{P}_{1}=\frac{D_{2} \partial_{z}-D_{3} \partial_{y}}{\partial^{2}}, \quad \hat{P}_{2}=\frac{D_{3} \partial_{x}-D_{1} \partial_{z}}{\partial^{2}},
$$




$$
\hat{P}_{3}=\frac{D_{1} \partial_{y}-D_{2} \partial_{x}}{\partial^{2}}+\widetilde{R}_{n} \cdot \frac{\partial_{z}\left(\widehat{D}_{T}-\frac{N_{A}}{L_{e}} \partial^{2}\right)}{\partial^{2} \hat{L}}+\widetilde{R a} \cdot \frac{\partial_{z}\left(\widehat{D}_{\varphi}+\frac{N_{B}}{L_{e}} \partial_{z}\right)}{\partial^{2} \hat{L}}
$$

and velocities: $v_{0}^{x}=u_{0}, v_{0}^{y}=v_{0}, v_{0}^{z}=w_{0}$. Using the formula (79), we can eliminate the pressure from the equation (74), and, as the result, we obtained a system of equations for finding the zero-order velocity field:

$$
\left\{\begin{array}{c}
\hat{d}_{11} u_{0}+\hat{d}_{12} v_{0}+\hat{d}_{13} w_{0}=\widehat{F}_{0}^{x} \\
\hat{d}_{21} u_{0}+\hat{d}_{22} v_{0}+\hat{d}_{23} w_{0}=F_{0}^{y} \\
\hat{d}_{31} u_{0}+\hat{d}_{32} v_{0}+\hat{d}_{33} w_{0}=0
\end{array}\right.
$$

The components of the tensor $\hat{d}_{i j}$ are

$$
\begin{gathered}
\hat{d}_{11}=\widehat{D}_{W}+\frac{D_{2} \partial_{x} \partial_{z}-D_{3} \partial_{x} \partial_{y}}{\partial^{2}}, \quad \hat{d}_{12}=\frac{D_{3} \partial_{x}^{2}-D_{1} \partial_{x} \partial_{z}}{\partial^{2}}-D_{3}, \\
\hat{d}_{13}=D_{2}+\frac{D_{1} \partial_{x} \partial_{y}-D_{2} \partial_{x}^{2}}{\partial^{2}}+\widetilde{R}_{n} \cdot \frac{\partial_{x} \partial_{z}\left(\widehat{D}_{T}-\frac{N_{A}}{L_{e}} \partial^{2}\right)}{\partial^{2} \hat{L}}+\widetilde{R a} \cdot \frac{\partial_{x} \partial_{z}\left(\widehat{D}_{\varphi}+\frac{N_{B}}{L_{e}} \partial_{z}\right)}{\partial^{2} \hat{L}}, \\
\hat{d}_{21}=D_{3}+\frac{D_{2} \partial_{y} \partial_{z}-D_{3} \partial_{y}^{2}}{\partial^{2}}, \quad \hat{d}_{22}=\widehat{D}_{W}+\frac{D_{3} \partial_{y} \partial_{x}-D_{1} \partial_{y} \partial_{z}}{\partial^{2}}, \\
\hat{d}_{23}=\widetilde{R}_{n} \cdot \frac{\partial_{y} \partial_{z}\left(\widehat{D}_{T}-\frac{N_{A}}{L_{e}} \partial^{2}\right)}{\partial^{2} \hat{L}}+\widetilde{R a} \cdot \frac{\partial_{y} \partial_{z}\left(\widehat{D}_{\varphi}+\frac{N_{B}}{L_{e}} \partial_{z}\right)}{\partial^{2} \hat{L}}+\frac{D_{1} \partial_{y}^{2}-D_{2} \partial_{y} \partial_{x}}{\partial^{2}}-D_{1}, \\
\hat{d}_{31}=\frac{D_{2} \partial_{z}^{2}-D_{3} \partial_{z} \partial_{y}}{\partial^{2}}-D_{2}, \quad \hat{d}_{32}=\frac{D_{3} \partial_{z} \partial_{x}-D_{1} \partial_{z}^{2}}{\partial^{2}}+D_{1}, \\
+\widetilde{R}_{n} \frac{\hat{d}_{z 3}^{2}\left(\widehat{D}_{T}-\frac{N_{A}}{L_{e}} \partial^{2}\right)}{\partial^{2} \hat{L}}+\frac{D_{1} \partial_{z} \partial_{y}-D_{2} \partial_{z} \partial_{x}+}{\partial^{2}}+\frac{\partial_{z}^{2}\left(\widehat{D}_{\varphi}+\frac{N_{B}}{L_{e}} \partial_{z}\right)}{\partial^{2} \hat{L}}-\widetilde{R}_{n} \cdot \frac{\left(\widehat{D}_{T}-\frac{N_{A}}{L_{e}} \partial^{2}\right)}{\hat{L}}-\widetilde{R a} \cdot \frac{\left(\widehat{D}_{\varphi}+\frac{N_{B}}{L_{e}} \partial_{z}\right)}{\hat{L}} .
\end{gathered}
$$

As it is known, the solution of the system of equations (80) is found by Cramer's rule:

$$
\begin{aligned}
& u_{0}=\frac{1}{\Delta}\left\{\left(\hat{d}_{22} \hat{d}_{33}-\hat{d}_{32} \hat{d}_{23}\right) F_{0}^{x}+\left(\hat{d}_{13} \hat{d}_{32}-\hat{d}_{12} \hat{d}_{33}\right) F_{0}^{y}\right\} \\
& v_{0}=\frac{1}{\Delta}\left\{\left(\hat{d}_{23} \hat{d}_{31}-\hat{d}_{21} \hat{d}_{33}\right) F_{0}^{x}+\left(\hat{d}_{11} \hat{d}_{33}-\hat{d}_{13} \hat{d}_{31}\right) F_{0}^{y}\right\} \\
& w_{0}=\frac{1}{\Delta}\left\{\left(\hat{d}_{21} \hat{d}_{32}-\hat{d}_{22} \hat{d}_{31}\right) F_{0}^{x}+\left(\hat{d}_{12} \hat{d}_{31}-\hat{d}_{11} \hat{d}_{32}\right) F_{0}^{y}\right\}
\end{aligned}
$$

Here, $\Delta$ is the determinant of the system of equations (80), which in an expanded form is

$$
\Delta=\hat{d}_{11} \hat{d}_{22} \hat{d}_{33}+\hat{d}_{21} \hat{d}_{32} \hat{d}_{13}+\hat{d}_{12} \hat{d}_{23} \hat{d}_{31}-\hat{d}_{13} \hat{d}_{22} \hat{d}_{31}-\hat{d}_{32} \hat{d}_{23} \hat{d}_{11}-\hat{d}_{21} \hat{d}_{12} \hat{d}_{33}
$$


Let us write the external force $\vec{F}_{0}$ in the complex form

$$
\vec{F}_{0}=\vec{i} \frac{f_{0}}{2} e^{i \varphi_{2}}+\vec{j} \frac{f_{0}}{2} e^{i \varphi_{1}}+\text { c.c. }
$$

Then all operators in formulae (81)-(84) act from the left on their eigenfunction:

$$
\begin{gathered}
\widehat{D}_{W, T, \varphi} e^{i \varphi_{1}}=e^{i \varphi_{1}} \widehat{D}_{W, T, \varphi}\left(\vec{k}_{0},-\omega_{0}\right)=e^{i \varphi_{1}} \widehat{D}_{W_{1}}^{*}, T_{1}, \varphi_{1}, \widehat{D}_{W, T, \varphi} e^{i \varphi_{2}}=e^{i \varphi_{2}} \widehat{D}_{W, T, \varphi}\left(\vec{k}_{0},-\omega_{0}\right)=e^{i \varphi_{2}} \widehat{D}_{W_{2}}^{*}, T_{2}, \varphi_{2}, \\
\Delta e^{i \varphi_{1}}=e^{i \varphi_{1}} \Delta\left(\vec{k}_{0},-\omega_{0}\right)=e^{i \varphi_{1}} \Delta_{1}^{*}, \quad \Delta e^{i \varphi_{2}}=e^{i \varphi_{2}} \Delta\left(\vec{k}_{0},-\omega_{0}\right)=e^{i \varphi_{2}} \Delta_{2}^{*},
\end{gathered}
$$

where the new notation is introduced

$$
\begin{gathered}
\widehat{D}_{W_{1}}^{*}=k_{0}^{2}-i \operatorname{Pr}^{-1}\left(\omega_{0}-k_{0} W_{1}\right), \quad \widehat{D}_{W_{2}}^{*}=k_{0}^{2}-i \operatorname{Pr}^{-1}\left(\omega_{0}-k_{0} W_{2}\right), \quad W_{1}=W_{-1}^{x}, \quad W_{2}=W_{-1}^{y}, \\
\widehat{D}_{T_{1}}^{*}=k_{0}^{2}-i\left(\omega_{0}-k_{0} W_{1}\right), \quad \widehat{D}_{T_{2}}^{*}=k_{0}^{2}-i\left(\omega_{0}-k_{0} W_{2}\right), \\
\widehat{D}_{\varphi_{1}}^{*}=L_{e}^{-1} k_{0}^{2}-i\left(\omega_{0}-k_{0} W_{1}\right), \quad \widehat{D}_{\varphi_{2}}^{*}=L_{e}^{-1} k_{0}^{2}-i\left(\omega_{0}-k_{0} W_{2}\right) . \\
\Delta_{1}^{*}=\widehat{D}_{W_{1}}^{*}\left(\widehat{D}_{W_{1}}^{*} \widehat{A}_{1}^{*}+D_{1}^{2}\right), \quad \Delta_{2}^{*}=\widehat{D}_{W_{2}}^{*}\left(\widehat{D}_{W_{2}}^{*} \widehat{A}_{2}^{*}+D_{2}^{2}\right) \\
\hat{A}_{1,2}^{*}=\widehat{D}_{W_{1,2}}^{*}-\widetilde{R}_{n} \cdot \frac{\widehat{D}_{T_{1,2}}^{*}+\frac{N_{A}}{L_{e}} k_{0}^{2}}{\hat{L}_{1,2}}-\frac{\widetilde{R a}_{D_{T_{1,2}}^{*}}}{\widetilde{R}_{1}^{*}}
\end{gathered}
$$

Here and below, we denote the complex-conjugate quantities by an asterisk. When performing the subsequent calculations, some of the components in the tensors $\hat{d}_{i j}$ become zero. Taking this fact into account, velocity fields of the zero approximation has the following form:

$$
\begin{gathered}
u_{0}=\frac{f_{0}}{2} \frac{\widehat{A}_{2}^{*}}{\hat{A}_{2}^{*} \widehat{D}_{W_{2}}^{*}+D_{2}^{2}} e^{i \varphi_{2}}+\text { c.c. }=u_{03}+u_{04} \\
v_{0}=\frac{f_{0}}{2} \frac{\widehat{D}_{1}^{*}}{\hat{A}_{1}^{*} \widehat{D}_{W_{1}}^{*}+D_{1}^{2}} e^{i \varphi_{1}}+\text { c.c. }=v_{01}+v_{02} \\
w_{0}=-\frac{f_{0}}{2} \frac{D_{1}}{\hat{A}_{1}^{*} \widehat{D}_{W_{1}}^{*}+D_{1}^{2}} e^{i \varphi_{1}}+\frac{f_{0}}{2} \frac{D_{2}}{\hat{A}_{2}^{*} \widehat{D}_{W_{2}}^{*}+D_{2}^{2}} e^{i \varphi_{2}}+\text { c.c. }=w_{01}+w_{02}+w_{03}+w_{04}
\end{gathered}
$$

It is easy to see that the component of the rotation parameter $D_{3}$ also drops out.

\section{CALCULATION OF THE REYNOLDS STRESSES}

To close the system of equations (31)-(32) that describe the evolution of the large-scale velocity fields $\vec{W}_{-1}$, it is necessary to calculate the following correlators:

$$
\begin{aligned}
& T^{31}=\overline{w_{0} u_{0}}=\overline{w_{01}\left(u_{01}\right)^{*}}+\overline{\left(w_{01}\right)^{*} u_{01}}+\overline{w_{03}\left(u_{03}\right)^{*}}+\overline{\left(w_{03}\right)^{*} u_{03}} \\
& T^{32}=\overline{w_{0} v_{0}}=\overline{w_{01}\left(v_{01}\right)^{*}}+\overline{\left(w_{01}\right)^{*} v_{01}}+\overline{w_{03}\left(v_{03}\right)^{*}}+\overline{\left(w_{03}\right)^{*} v_{03}}
\end{aligned}
$$

Substituting the solutions for the small-scale velocity fields (87)-(89) obtained in Appendix B, into the equations (90)-(91), we can find the following expression for the correlators: 


$$
\begin{gathered}
T^{31}=\frac{f_{0}^{2}}{4} \frac{D_{2}\left(\hat{A}_{2}+\hat{A}_{2}^{*}\right)}{\left|\hat{A}_{2} \widehat{D}_{W_{2}}+D_{2}^{2}\right|^{2}} \\
T^{32}=-\frac{f_{0}^{2}}{4} \frac{D_{1}\left(\hat{A}_{1}+\hat{A}_{1}^{*}\right)}{\left|\hat{A}_{1} \widehat{D}_{W_{1}}+D_{1}^{2}\right|^{2}}
\end{gathered}
$$

Then with the definition of the operators $\widehat{D}_{W_{1,2}}$ and $\hat{A}_{1,2}$, we can write down the series of useful relations for the calculation of $T^{31}$ and $T^{32}$ :

$$
\begin{aligned}
& \left|\widehat{D}_{W_{1,2}}\right|^{2}=\widehat{D}_{W_{1,2}} \widehat{D}_{W_{1,2}}^{*}=k_{0}^{4}+\operatorname{Pr}^{-2} \widetilde{\omega}_{1,2}^{2}, \quad\left|\widehat{D}_{T_{1,2}}\right|^{2}=\widehat{D}_{T_{1,2}} \widehat{D}_{T_{1,2}}^{*}=k_{0}^{4}+\widetilde{\omega}_{1,2}^{2}, \\
& \left|\widehat{D}_{\varphi_{1,2}}\right|^{2}=\widehat{D}_{\varphi_{1,2}} \widehat{D}_{\varphi_{1,2}}^{*}=L_{e}^{-2} k_{0}^{4}+\widetilde{\omega}_{1,2}^{2}, \quad \widetilde{\omega}_{1,2}=\omega_{0}-k_{0} W_{1,2} \\
& \left|\hat{A}_{1,2}\right|^{2}=\hat{A}_{1,2} \hat{A}_{1,2}^{*}=k_{0}^{4}+\operatorname{Pr}^{-2} \widetilde{\omega}_{1,2}^{2}-2 \widetilde{R a} \cdot \frac{k_{0}^{4}-\operatorname{Pr}^{-1} \widetilde{\omega}_{1,2}^{2}}{k_{0}^{4}+\widetilde{\omega}_{1,2}^{2}}+\frac{\widetilde{R a}^{2}}{k_{0}^{4}+\widetilde{\omega}_{1,2}^{2}}+r_{n_{1,2}}, \\
& r_{n_{1,2}}=-2 \widetilde{R}_{n} \cdot \frac{\left(k_{0}^{4}+\widetilde{\omega}_{1,2}^{2}\right)\left(L_{e}^{-1} k_{0}^{4}-\operatorname{Pr}^{-1} \widetilde{\omega}_{1,2}^{2}\right)+\frac{N_{A}}{L_{e}} k_{0}^{2}\left(L_{e}^{-1} k_{0}^{2}-\operatorname{Pr}^{-1} \widetilde{\omega}_{1,2}^{2} k_{0}^{2}-\widetilde{\omega}_{1,2}^{2} k_{0}^{2}\left(1+\operatorname{Pr}^{-1} L_{e}^{-1}\right)\right)}{\left(k_{0}^{4}+\widetilde{\omega}_{1,2}^{2}\right)\left(L_{e}^{-2} k_{0}^{4}+\widetilde{\omega}_{1,2}^{2}\right)}+ \\
& +\widetilde{R}_{n}^{2} \cdot \frac{k_{0}^{4}\left(1+\frac{2 N_{A}}{L_{e}}+\frac{N_{A}^{2}}{L_{e}^{2}}\right)+\widetilde{\omega}_{1,2}^{2}}{\left(k_{0}^{4}+\widetilde{\omega}_{1,2}^{2}\right)\left(L_{e}^{-2} k_{0}^{4}+\widetilde{\omega}_{1,2}^{2}\right)}+2 \widetilde{R}_{n} \widetilde{R a} \cdot \frac{\widetilde{\omega}_{1,2}^{2}+k_{0}^{2} L_{e}^{-1}\left(1+\frac{N_{A}}{L_{e}}\right)}{\left(k_{0}^{4}+\widetilde{\omega}_{1,2}^{2}\right)\left(L_{e}^{-2} k_{0}^{4}+\widetilde{\omega}_{1,2}^{2}\right)}, \\
& \widehat{D}_{W_{1,2}} \hat{A}_{1,2}+\widehat{D}_{W_{1,2}}^{*} \hat{A}_{1,2}^{*}=2\left(k_{0}^{4}-\operatorname{Pr}^{-2} \widetilde{\omega}_{1,2}^{2}\right)-2 \widetilde{R a} \cdot \frac{k_{0}^{4}+\operatorname{Pr}^{-1} \widetilde{\omega}_{1,2}^{2}}{k_{0}^{4}+\widetilde{\omega}_{1,2}^{2}}- \\
& -2 \widetilde{R}_{n} \cdot \frac{\left(k_{0}^{4}+\widetilde{\omega}_{1,2}^{2}\right)\left(L_{e}^{-1} k_{0}^{4}+\operatorname{Pr}^{-1} \widetilde{\omega}_{1,2}^{2}\right)+\frac{N_{A}}{L_{e}} k_{0}^{4}\left(L_{e}^{-1} k_{0}^{4}+\operatorname{Pr}^{-1} \widetilde{\omega}_{1,2}^{2}+\widetilde{\omega}_{1,2}^{2}\left(\operatorname{Pr}^{-1} L_{e}^{-1}-1\right)\right)}{\left(k_{0}^{4}+\widetilde{\omega}_{1,2}^{2}\right)\left(L_{e}^{-2} k_{0}^{4}+\widetilde{\omega}_{1,2}^{2}\right)} .
\end{aligned}
$$

Using these relations, we can obtain the following expressions:

$$
\begin{aligned}
& \hat{A}_{1,2}+\hat{A}_{1,2}^{*}=2\left(k_{0}^{2}-\frac{\widetilde{R a} k_{0}^{2}}{k_{0}^{4}+\widetilde{\omega}_{1,2}^{2}}-\widetilde{R}_{n} \cdot \frac{L_{e}^{-1} k_{0}^{2}+\frac{N_{A}}{L_{e}}\left(L_{e}^{-1} k_{0}^{4}-\widetilde{\omega}_{1,2}^{2}\right)}{L_{e}^{-2} k_{0}^{4}+\widetilde{\omega}_{1,2}^{2}}\right), \\
& \left|\widehat{D}_{W_{1,2}} \widehat{A}_{1,2}+D_{1,2}^{2}\right|^{2}= \\
& =\left(k_{0}^{4}+\operatorname{Pr}^{-2} \widetilde{\omega}_{1,2}^{2}\right)\left(k_{0}^{4}+\operatorname{Pr}^{-2} \widetilde{\omega}_{1,2}^{2}+\frac{\widetilde{R a}^{2}}{k_{0}^{4}+\widetilde{\omega}_{1,2}^{2}}-2 \widetilde{R a} \cdot \frac{k_{0}^{4}-\operatorname{Pr}^{-1} \widetilde{\omega}_{1,2}^{2}}{k_{0}^{4}+\widetilde{\omega}_{1,2}^{2}}+r_{n_{1,2}}\right)+ \\
& +2 D_{1,2}^{2}\left(k_{0}^{4}-\operatorname{Pr}^{-2} \widetilde{\omega}_{1,2}^{2}-\widetilde{R a} \cdot \frac{k_{0}^{4}+\operatorname{Pr}^{-1} \widetilde{\omega}_{1,2}^{2}}{k_{0}^{4}+\widetilde{\omega}_{1,2}^{2}}+p_{n_{1,2}}\right)+D_{1,2}^{4}, \\
& p_{n_{1,2}}=-\widetilde{R}_{n} \cdot \frac{\left(k_{0}^{4}+\widetilde{\omega}_{1,2}^{2}\right)\left(L_{e}^{-1} k_{0}^{4}+\operatorname{Pr}^{-1} \widetilde{\omega}_{1,2}^{2}\right)+\frac{N_{A}}{L_{e}} k_{0}^{4}\left(L_{e}^{-1} k_{0}^{4}+\operatorname{Pr}^{-1} \widetilde{\omega}_{1,2}^{2}+\widetilde{\omega}_{1,2}^{2}\left(\operatorname{Pr}^{-1} L_{e}^{-1}-1\right)\right)}{\left(k_{0}^{4}+\widetilde{\omega}_{1,2}^{2}\right)\left(L_{e}^{-2} k_{0}^{4}+\widetilde{\omega}_{1,2}^{2}\right)} .
\end{aligned}
$$


Substituting (94) in (92)-(93) we can find expressions for the Reynolds stresses in the general form:

$$
\begin{aligned}
& T^{31}=\frac{f_{0}^{2}}{2} \frac{D_{2} k_{0}^{2}\left(k_{0}^{4}+\widetilde{\omega}_{2}^{2}-\widetilde{R a}-l_{n_{2}}\right)}{\left(k_{0}^{4}+\widetilde{\omega}_{2}^{2}\right) \Lambda_{2}}, \\
& T^{32}=-\frac{f_{0}^{2}}{2} \frac{D_{1} k_{0}^{2}\left(k_{0}^{4}+\widetilde{\omega}_{2}^{2}-\widetilde{R a}-l_{n_{1}}\right)}{\left(k_{0}^{4}+\widetilde{\omega}_{1}^{2}\right) \Lambda_{1}},
\end{aligned}
$$

where $\Lambda_{1,2}=\left|\widehat{D}_{W_{1,2}} \hat{A}_{1,2}+D_{1,2}^{2}\right|^{2}$. Expressions for $l_{n_{1,2}}$ are:

$$
l_{n_{1,2}}=\widetilde{R}_{n} \cdot \frac{L_{e}^{-1}+\frac{N_{A}}{L_{e}}\left(k_{0}^{2} L_{e}^{-1}-\frac{\tilde{\omega}_{1,2}^{2}}{k_{0}^{2}}\right)}{L_{e}^{-2} k_{0}^{4}+\widetilde{\omega}_{1,2}^{2}} \cdot\left(k_{0}^{4}+\widetilde{\omega}_{1,2}^{2}\right) .
$$

If the Prandtl number of the nanofluid is approximately equal to one $\operatorname{Pr}=1$, then the expressions for the components of the Reynolds stresses are simplified:

$$
\begin{gathered}
T^{31}=\frac{f_{0}^{2} D_{2} k_{0}^{2}\left(k_{0}^{4}+\widetilde{\omega}_{2}^{2}-\widetilde{R a}-l_{n_{2}}\right)}{2\left(k_{0}^{4}+\widetilde{\omega}_{2}^{2}\right)\left(\left(k_{0}^{4}+\widetilde{\omega}_{2}^{2}\right)^{2}+2\left(D_{2}^{2}-\widetilde{R a}\right)\left(k_{0}^{4}-\widetilde{\omega}_{2}^{2}\right)+\left(D_{2}^{2}-\widetilde{R a}\right)^{2}+r_{n_{2}}\left(k_{0}^{4}+\widetilde{\omega}_{2}^{2}\right)+2 p_{n_{2}} D_{2}^{2}\right)}, \\
T^{32}=-\frac{f_{0}^{2} D_{1} k_{0}^{2}\left(k_{0}^{4}+\widetilde{\omega}_{1}^{2}-\widetilde{R a}-l_{n_{1}}\right)}{2\left(k_{0}^{4}+\widetilde{\omega}_{1}^{2}\right)\left(\left(k_{0}^{4}+\widetilde{\omega}_{1}^{2}\right)^{2}+2\left(D_{1}^{2}-\widetilde{R a}\right)\left(k_{0}^{4}-\widetilde{\omega}_{1}^{2}\right)+\left(D_{1}^{2}-\widetilde{R a}\right)^{2}+r_{n_{1}}\left(k_{0}^{4}+\widetilde{\omega}_{1}^{2}\right)+2 p_{n_{1}} D_{1}^{2}\right)} .
\end{gathered}
$$

Here the values of the coefficients $r_{n_{1,2}}$ and $p_{n_{1,2}}$ are taken with $\operatorname{Pr}=1$.

\section{ORCID IDs}

(D)Michael I. Kopp, https://orcid.org/0000-0001-7457-3272; (DAnatoly V. Tur, https://orcid.org/0000-0002-3889-8130, (D)Volodymyr V. Yanovsky, https://orcid.org/0000-0003-0461-749X

\section{REFERENCES}

[1] S.K. Das, S. U.S Choi, W.Yu, and T. Pradeep, Nanofluids: science and technology, (Hoboken, New Jersey, Wiley-Interscience, 2008), pp. 397.

[2] Dhananjay Yadav, G.S. Agrawal, and R. Bhargava, International Journal of Engineering Science, 49, 1171-1184 (2011), https://doi.org/10.1016/j.ijengsci.2011.07.002

[3] Shilpi Agarwal, and B.S. Bhadauria, Continuum Mech. Thermodyn., 26, 437-445 (2014), https://doi.org/10.1007/s00161-0130309-6

[4] R. Chand, G.C. Rana, and S. K. Kango, FME Transactions, 43, $62-69$ (2015), https://scindeks.ceon.rs/article.aspx?artid=1451$20921501062 \mathrm{C}$

[5] B.L. Smorodin, S.M. Ishutov, and B.I. Myznikova, Microgravity Science and Technology, 30, 95-102 (2018), https://doi.org/10.1007/s12217-017-9582-5

[6] M.I. Kopp, A.V. Tur, and V.V. Yanovsky, https://arxiv.org/abs/1706.00223v1

[7] U. Frisch, Z.S. She, and P.L. Sulem, Physica D, 28, 382-392 (1987), https://doi.org/10.1016/0167-2789(87)90026-1

[8] M.I. Kopp, A.V. Tur, and V.V Yanovsky, Open Journal of Fluid Dynamics, 05(04), 311-321 (2015), https://doi.org/10.4236/ojfd.2015.54032

[9] G. Rüdiger, Astron. Nachr. 299(4), 217-222 (1978), https://doi.org/10.1002/asna.19782990408.

\section{ВИХРОВЕ ДИНАМО В СТРАТИФІКОВАНІЙ НАНОРІДИНІ, ЩО ПОХИЛО ОБЕРТАЕТЬСЯ 3 ДРІБНОМАСШТАБНОЮ НЕСПІРАЛЬНОЮ СИЛОЮ \\ Михайло Й. Копп ${ }^{\text {, Анатолій В. Тур }}$, Володимир В. Яновський ${ }^{a, b}$ \\ анститут монокристалів, Національна Академія Наук Украӥни пр. Науки 60, 61001 Харків, Украӥна \\ ${ }^{b}$ Харківський національний університет імені В.Н. Каразина майдан Свободи, 4, 61022, Харків, Украӥна \\ ${ }^{c}$ Universite Toulouse [UPS], CNRS, Institute of Research for Astrophysics and Planetology 9 avenue du Colonel Roche, BP 44346, 31028 Toulouse Cedex 4, France}

В роботі отримана великомасштабна нестійкість гідродинамічного $\alpha$-ефекту в стратифікованій нанорідині, що похило обертається, з урахуванням ефектів броунівської дифузії і потоку частинок під дією градієнта температури (термофорезу). Нестійкість викликається дією зовнішньої дрібномасштабної неспіральної сили, яка збуджує дрібномасштабні коливання 
швидкості з нульовою спіральністю і малим числом Рейнольдса. Нелінійні рівняння для великомасштабних рухів отримані 3 використанням методу багатомасштабних асимптотичних розкладів за малим параметром (числом Рейнольдса). Досліджена лінійна великомасштабна нестійкість типу гідродинамічного $\alpha$-ефекту в залежності від параметрів обертання $D$, температурної стратифікації $\widetilde{R a}$ і концентрації наночастинок $\widetilde{R}_{n}$. Отриманий новий ефект генерації великомасштабних вихрових структур в нанорідині при $\widetilde{R a}=0$, пов'язаний зі збільшенням концентрації наночастинок. Максимальний інкремент нестійкості досягається при кутах нахилу $\theta \approx \pi / 5$ для чисел Прандтля $\operatorname{Pr}=5$, а для чисел Прандтля $\operatorname{Pr}=1$ при кутах нахилу $\theta \approx \pi / 2$. Встановлено, що зміна частоти параметричного впливу дозволить контролювати і відслідковувати процес генерації великомасштабних вихрових структур. Показано, що циркулярно поляризовані вихори Бельтрамі виникають в нанорідині в результаті розвитку нової великомасштабної нестійкості. В роботі досліджується режим насичення великомасштабної нестійкості в стратифікованій нанорідині, що похило обертається 3 зовнішньою дрібномасштабною неспіральною силою. У стаціонарному режимі була отримана динамічна система рівнянь для великомасштабних збурень поля швидкості. Отримані чисельні рішення цієї системи рівнянь, які показують існування локалізованих вихрових структур у вигляді нелінійних хвиль Бельтрамі і кінків. Профіль швидкості кінка має тенденцію бути постійним при великих значеннях Z.

КЛЮЧОВІ СЛОВА: стратифікована нанорідина, великомасштабна нестійкість, сила Коріоліса, багатомасштабні асимптотичні розкладання, $\alpha$-эфект, локалізовані вихрові структури 